\title{
PI3K/Akt Pathway Contributes to Neurovascular Unit Protection of Xiao-Xu-Ming Decoction against Focal Cerebral Ischemia and Reperfusion Injury in Rats
}

\author{
Rui Lan, ${ }^{1}$ Jun Xiang, ${ }^{1,2}$ Yong Zhang, ${ }^{3}$ Guo-Hua Wang, ${ }^{1}$ Jie Bao, ${ }^{4}$ Wen-Wei Li, ${ }^{1}$ Wen Zhang, \\ Li-Li Xu, ${ }^{1}$ and Ding-Fang Cai ${ }^{1}$ \\ ${ }^{1}$ Department of Integrative Medicine, Zhongshan Hospital, and Laboratory of Neurology, Institute of Integrative Medicine, \\ Fudan University, Shanghai 200032, China \\ ${ }^{2}$ Longhua Hospital, Shanghai University of Traditional Chinese Medicine, Shanghai 200032, China \\ ${ }^{3}$ Guangzhou University of Traditional Chinese Medicine, Guangzhou 510006, China \\ ${ }^{4}$ Shuguang Hospital, Shanghai University of Traditional Chinese Medicine, Shanghai 200021, China
}

Correspondence should be addressed to Ding-Fang Cai; dingfangcai@163.com

Received 7 February 2013; Revised 31 March 2013; Accepted 9 April 2013

Academic Editor: Roman Huber

Copyright (C) 2013 Rui Lan et al. This is an open access article distributed under the Creative Commons Attribution License, which permits unrestricted use, distribution, and reproduction in any medium, provided the original work is properly cited.

\begin{abstract}
In the present study, we used a focal cerebral ischemia and reperfusion rat model to investigate the protective effects of $X i a o-X u$ Ming decoction (XXMD) on neurovascular unit and to examine the role of PI3K (phosphatidylinositol 3-kinase)/Akt pathway in this protection. The cerebral ischemia was induced by $90 \mathrm{~min}$ of middle cerebral artery occlusion. Cerebral infarct area was measured by tetrazolium staining, and neurological function was observed at $24 \mathrm{~h}$ after reperfusion. DNA fragmentation assay, combined with immunofluorescence, was performed to evaluate apoptosis of neuron, astrocyte, and vascular endothelial cell which constitute neurovascular unit. The expression levels of proteins involved in PI3K/Akt pathway were detected by Western blot. The results showed that XXMD improved neurological function, decreased cerebral infarct area and neuronal damage, and attenuated cellular apoptosis in neurovascular unit, while these effects were abolished by inhibition of PI3K/Akt with LY294002. We also found that XXMD upregulated p-PDKl, p-Akt, and p-GSK3 $\beta$ expression levels, which were partly reversed by LY294002. In addition, the increases of p-PTEN and p-c-Raf expression levels on which LY294002 had no effect were also observed in response to XXMD treatment. The data indicated the protective effects of XXMD on neurovascular unit partly through the activation of PI3K/Akt pathway.
\end{abstract}

\section{Introduction}

Current biomedical research about stroke is focusing on treating neurovascular unit (NVU), and it is widely accepted that the key to effective therapy lies in restoring normal function of NVU.

NVU is a functionally and structurally interdependent multicellular complex which consists of endothelial cells, basal lamina, pericytes, astrocytes, and neurons [1], and the various components of NVU dynamically interact and act as an intricate network to maintain the homeostatic microenvironment for neuronal survival and function [2]. Amounting evidence indicates that NVU plays an important role in physiological functions and pathogenesis of many central nervous system diseases such as stroke and Alzhimer's disease $[2,3]$. Despite a growing comprehension of the molecular process that causes ischemia injury to neuron, we still need to understand the whole changes of NUV after stroke such as glial cells and endothelial cells, not only neurons.

Cerebrovasculature and parenchymal tissues are involved in the pathogenesis of stroke through the active interaction of multiple mechanisms, and ischemic penumbra as the potentially therapeutic target has become the focal point of stroke research. Apoptosis, a special form of cell death, occurs in this highly complex process, and neuronal fate 
after ischemia is dependent on the balance between apoptotic and survival signals. Evidence has been presented that apoptosis appears in the peripheral penumbra of ischemia $[4,5]$ and PI3K/Akt pathway mediates neuronal survival after cerebral ischemia and reperfusion [6-8]. Phosphorylation of Akt promotes cell survival against cerebral ischemic insult by phosphorylation and subsequent inactivation of many proapoptotic proteins, such as glycogen synthase kinse $3 \beta$ (GSK3 $\beta$ ) [7], Bad [9], and Forkhead transcription factors [10]. Although there is growing evidence that PI3K/Akt pathway and neuronal survival following cerebral ischemia are closely correlated, few studies further clarify whether $\mathrm{PI} 3 \mathrm{~K} / \mathrm{Akt}$ pathway contributes to the protection of NVU after cerebral ischemia and reperfusion.

To date, a large body of basic studies have indicated that numerous agents or options could reduce cerebral infarct area and improve neurological deficits with animal models of stroke [11-21] and the suggested mechanisms may be associated with suppression of apoptosis $[13-15,19]$, inflammation $[16,17,19]$, glutamate excitotoxicity $[18,21]$, energy deficiency $[11,20]$, superoxide-mediated oxidative stress [12], and promotion of angiogenesis [19] by activation of signal pathways. However, few potential drugs or strategies could eventually be applied to benefit patients with stroke. A report summarized the problems which may cause the failures of the conversion and showed that there were many discrepancies on the outcome measures, functional assessment, and discrepancies of premorbid conditions, therapeutic windows, and drugdosing schedules between preclinical and clinical research [22]. Facing these successes and failures, more advances in the comprehension of the pathophysiology of stroke and more exploration of novel agents are needed [22], especially when the critical role of NVU in cerebral ischemia is proposed.

Traditional Chinese medicine (TCM) which is derived from natural plants and animals may act on multiple targets, and its flexible and comprehensive treatment strategies usually generate unexpected outcomes. These characteristics of TCM coincide with the view of NVU protection. Based on this fact, TCM has become the focus of medical research [23-27]. Xiao-Xu-Ming decoction (XXMD), one classic TCM formula recorded by Sun Simiao of the Chinese ancient Tang Dynasty, has been widely used in the treatment of stroke. It has aroused extensively preclinical and clinical studies for its high efficacy [28-32]. Lines of evidence have shown that active components of XXMD could reduce cerebral infarct area, improve behavioral test, decrease iNOS activity, and attenuate mitochondrial dysfunction after cerebral ischemia $[29,30]$. A recent study has reported that oral administration of middle $(30 \mathrm{~g} / \mathrm{kg} /$ day $)$ and high $(60 \mathrm{~g} / \mathrm{kg} /$ day $)$ dosages of XXMD could significantly inhibit apoptotic neuronal death and improve the spatial cognitive performances after ischemia. Besides, the serum isolated from XXMD-treated $(60 \mathrm{~g} / \mathrm{kg} /$ day $)$ rats could block neuronal death in OGD model [28]. Most importantly, it has been proved that XXMD treatment could improve neurological outcome of patients with stroke in clinical research $[31,32]$. However, NVU protection of XXMD has not been explored. Thus, we determined to identify whether XXMD could exert protective effects on
NUV and confirm the role of PI3K/Akt pathway in NUV protection following cerebral ischemia and reperfusion.

\section{Materials and Methods}

2.1. Reagents. LY294002 (PI3K inhibitor), the primary antibodies for phospho-PDK1 (p-PDK1, Ser241), total PDK1, phospho-PTEN (p-PTEN, Ser380), total PTEN, phospho-Akt (p-Akt, Ser473), total Akt, phospho-c-Raf (p-c-Raf, Ser259), total c-Raf, phospho-GSK3 $\beta$ (p-GSK3 $\beta$, Ser9), total GSK3 $\beta$, GAPDH, and horseradish-peroxidase- (HRP-) linked antirabbit antibody purchased from Cell Signaling Technology (Beverly, MA, USA) were used for Western blot analysis. Mouse monoclonal antibodies for NeuN and GFAP from Millipore (Billerica, MA, USA), CD31 from Abcam (HK, China), and Alexa Fluor 555 anti-mouse antibody from life technologies (Carlsbad, CA, USA) were applied to immunofluorescence.

2.2. Preparation of XXMD. The XXMD consists of twelve crude drugs including Herba Ephedrae, cassia twig, Radix Paeoniae Alba, Rhizome Chuanxiong, Radix Ginseng, Radix Stephaniae Tetrandrae, Radix Scutellariae, Semen Armeniacae Amarum, Radix Aconiti Praeparata, Radix Glycyrrhizae, Radix Ledebouriellae, and fresh Rhizoma Zingiberis Recens in a ratio of $1: 1: 1: 1: 1: 1: 1: 1: 1: 1: 1.5: 5$. The crude drugs were purchased from Traditional Chinese Medicine Pharmacy of Zhongshan Hospital, Fudan University. XXMD was prepared as previously described with small modifications [28]. After the first decoction, conducted for $1 \mathrm{~h}$ in a $1: 10$ $(\mathrm{w} / \mathrm{v})$ drugs : water ratio, the suspension was filtered. Water was added for the second decoction, duration of about $1 \mathrm{~h}$, followed by the third time which lasted $1 \mathrm{~h}$. The gruffs were soaked in $75 \%$ ethanol for $24 \mathrm{~h}$ and the liquid was preserved. The filtered and mixed suspension from three decoctions was collected and centrifuged at $2000 \times \mathrm{g}$ for $20 \mathrm{~min}$ to obtain a suspension for the following preparation. Ethanol was added slowly with fast agitation until the concentration reached $75 \%$ ethanol (v/v). The suspension and the liquid acquired from the gruffs were merged and centrifuged at $2000 \times \mathrm{g}$ for $20 \mathrm{~min}$ then concentrated at the final concentration of $2 \mathrm{~g} / \mathrm{mL}(\mathrm{w} / \mathrm{v})$. The ethanol was recovered simultaneously with a rotary evaporator. Eventually, the liquid was autoclaved and stored at $-20^{\circ} \mathrm{C}$ before administration.

2.3. Animals and Drug Administration. One hundred and six male Sprague-Dawley rats weighing 250-280 g (Experimental Animal Center, Zhongshan Hospital, Fudan University, China) were housed in groups of four with free access to food and water and maintained in temperature $\left(22 \pm 2^{\circ} \mathrm{C}\right)$ and humidity-controlled ( $55 \pm 5 \%$ ) room with 12:12 h light-dark cycle. Prior to experimental manipulation, rats were handled daily for 3 days.

We usually apply the water extract of XXMD in humans and the common human daily dosage of XXMD is $165 \mathrm{~g} / 75 \mathrm{~kg}$ in body weight. According to the formula, $d_{\text {rat }}=d_{\text {human }} \times$ $0.71 / 0.11$ [33], the common dosage of XXMD in rat should be $14.2 \mathrm{~g} / \mathrm{kg} /$ day. As the rat's drug tolerance is higher than 
that of human [33], we therefore selected 15, 30, and $60 \mathrm{~g} / \mathrm{kg} / \mathrm{day}$ as low, medium, and high dosages, respectively, to investigate the effects of different dosages of XXMD on focal cerebral ischemia and reperfusion injury in the preliminary experiments. Comparing all results, we finally chose $60 \mathrm{~g} / \mathrm{kg} / \mathrm{day}$, as the optimal dose, to further investigate the underlying mechanisms, because it is better than others in drug effect which is probably due to the higher concentration of bioactivity of XXMD at a high dosage. Moreover, the dosages were referred to previous study [28]. In the study, rats were randomly divided into five groups: sham control (Sham) group, ischemia and reperfusion (I/R) group, ischemia and reperfusion plus XXMD $(60 \mathrm{~g} / \mathrm{kg} / \mathrm{day}$, XXMD60) group, ischemia and reperfusion plus XXMD $(60 \mathrm{~g} / \mathrm{kg} /$ day), and LY294002 (XXMD60 + LY294002) group, ischemia and reperfusion plus XXMD (60 g/kg/day), and the solution of dimethyl sulfoxide and ethanol (XXMD60 + vehicle) group.

For drug treatment, the rats in XXMD-treated groups were orally administered with XXMD $(60 \mathrm{~g} / \mathrm{kg} /$ day $)$ and others in the Sham, I/R groups were given the same volume of distilled water for 3 days before cerebral ischemic surgery. The drug administrations were performed twice a day at 8:00 and 18:00 and continued until animals were sacrificed. The above procedures were carried out by one investigator who was aware of the animal grouping but did not participate in the sequent investigations. All experimental protocols and animal handling procedures were approved by the Animal Care and Use Committee (ACUC) of Fudan University, and were consistent with the National Institutes of Health Guide for the Care and Use of laboratory Animals.

2.4. Focal Cerebral Ischemia and Reperfusion. Focal cerebral ischemia and reperfusion were performed as described previously [34]. Briefly, rats were anesthetized with $10 \%$ chloral hydrate $(350 \mathrm{mg} / \mathrm{kg}$, intraperitoneal, i.p.), and the left common carotid artery (CCA), the external carotid artery (ECA), and the internal carotid artery (ICA) were exposed. A length of 3.0 monofilament nylon suture (18.5-19.5 mm) with its rounded tip was advanced from the ECA into the lumen of the ICA until it blocked the origin of the middle cerebral artery (MCA). Reperfusion was initiated by withdrawal of the suture until the tip cleared the lumen of the ECA after 90 min of occlusion. In the present study, the rats in the $\mathrm{I} / \mathrm{R}$ and XXMD-treated groups were subjected to cerebral ischemia followed by reperfusion. Sham-operated animals underwent the same surgical procedure, but the suture was not introduced.

It should be noted that there are several technical factors in the MCAO rat model which may affect cerebral infarct area, such as physical differences in the employed monofilament suture, the depth of the insertion, and accidental premature reperfusion [35-38]. In order to avoid the occurrence of the above technical factors, the employed monofilament sutures were unified and purchased from Beijing Sunbio Biotech Co., Ltd. (Beijing, China). One adequately trained operator who was blind to the experimental design performed the focal cerebral ischemia and reperfusion induction

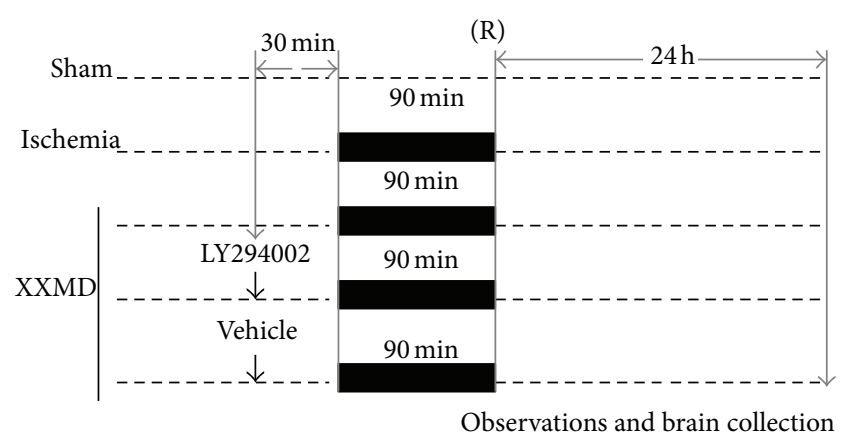

FIGURE 1: Schematic representation of the experimental protocols. Rats were randomly divided into five groups. Sham group in which the left ICA was exposed surgically but not subjected to occlusion. In the I/R and XXMD-treated groups, focal cerebral ischemia was induced in rats by MCAO for $90 \mathrm{~min}$ followed by reperfusion for $24 \mathrm{~h}$ as indicated in this figure. In the XXMD-treated group, the rats were administered with XXMD for 3 days before MCAO. In some cases, XXMD-treated rats received by intracerebroventricular injection of either the PI3K inhibitor LY294002 or vehicle $30 \mathrm{~min}$ prior to ischemic induction. As shown in this figure, neurological functions were observed and brains were collected at $24 \mathrm{~h}$ after reperfusion for TTC staining, histological analyses, TUNEL staining, immunofluorescence staining, and Western blot. Sham, sham control; ICA, internal carotid artery; I/R, ischemia and reperfusion; $\mathrm{XXMD}$, Xiao-Xu-Ming decoction; MCAO, middle cerebral artery occlusion; $\mathrm{R}$, reperfusion after cerebral ischemia.

using the standardized surgical and technical procedures to generate reproducible ischemic lesions in animal experiments.

2.5. Intracerebral Ventricular Injection. To investigate the function of PI3K after cerebral ischemia and reperfusion, several rats in XXMD-treated group were pretreated with LY294002, the PI3K special inhibitor, as described previously [39] with small modifications. LY294002 was dissolved in dimethyl sulfoxide (DMSO) and ethanol (ETOH) to make a final concentration of $10 \mathrm{mM}$ before the operation. The preparation of LY294002 and vehicle was performed by the same investigator who was responsible for the drug treatments. Rats were securely placed into the stereotaxic device under $10 \%$ chloral hydrate $(350 \mathrm{mg} / \mathrm{kg}$, i.p.) anesthesia with bregma and lambda at a horizontal level. The skulls were exposed and determined the coordinates: anteroposterior (AP): $-0.8 \mathrm{~mm}$ from bregma; mediolateral (ML): $1.4 \mathrm{~mm}$ from midline in the left side; dorsoventral (DV): $-3.6 \mathrm{~mm}$ from skull, adapted from Paxinos and Watson [40]. A $10 \mu \mathrm{L}$ of LY294002 solution or the small amount of vehicle (DMAO + ETOH) was injected intracerebroventricularly on the ischemic side at 30 min before operation. The experimental design for the present study was shown in Figure 1.

2.6. Neurological Deficits Score. Examination of neurological deficits was performed at $24 \mathrm{~h}$ after reperfusion by one investigator who was unaware of the experiment design. The neurological deficits were scored on a four-point scale described by Hara et al. [41] with a minor modification as 
follows: 0 indicated no neurological deficit; 1, mild focal neurological deficit (animal showed forelimb flexion); 2, moderate focal neurological deficit (decreased resistance to lateral push and forelimb flexion without circling); 3, severe focal deficit (decreased resistance to lateral push and forelimb flexion with circling). In the present study, the rats subjected to MCAO without any detectable neurological deficits were excluded from the following investigations and analyses to exclude operative failures.

2.7. Cerebral Infarct Area. Cerebral infarct area was measured by 2,3,5-triphenyl tetrazolium chloride (TTC; Sigma-Aldrich, St. Louis, MO, USA) staining. Rats were killed at $24 \mathrm{~h}$ after reperfusion and perfused transcardially with normal saline. Brains were sectioned into six coronal slices from rostal to caudal and stained with $1 \%$ TTC staining at $37^{\circ} \mathrm{C}$ for $20 \mathrm{~min}$ away from light. Then the brain tissues were differentiated according to white-colored infarct area and redpurple noninfarct area. Cerebral infarct areas were calculated using microscope image-analysis software (Image-Proplus, USA) according to the following formula: (contralateral hemisphere area - (ipsilateral hemisphere area - infarct area)/contralateral hemisphere area) $\times 100 \%$.

2.8. General Histology. At $24 \mathrm{~h}$ after reperfusion, the rats were deep anesthetized with $10 \%$ chloral hydrate $(350 \mathrm{mg} / \mathrm{kg}$, i.p.) and perfused transcardially with $200 \mathrm{~mL}$ normal saline followed by $4 \%$ paraformaldehyde $(0.1 \mathrm{M}$ phosphate buffered saline, $\mathrm{pH}$ 7.4). The brains were removed and postfixed in the same fixative for $24 \mathrm{~h}$ at $4^{\circ} \mathrm{C}$. After dehydration in graded ethanol and xylene, the brains were embedded in paraffin and sectioned into slices of $5 \mu \mathrm{m}$ on a rotary microtome. To assess brain injury, Nissl staining was performed with toluidine blue. Briefly, slices were dewaxed, dehydrated, and stained with $1 \%$ toluidine blue (Sigma-Aldrich, St. Louis, MO, USA) at $50^{\circ} \mathrm{C}$ for $30 \mathrm{~min}$. Then the slices were rinsed and cleared in graded ethanol and xylene and coverslipped under permount. The photomicrographs were captured with a light microscope. And the number of intact cells in penumbra of ischemic cortex was counted throughout five lesion regions randomly.

2.9. Immunofluorescence and TUNEL Staining. In order to detect the extent of DNA fragmentation of different cells in NVU, double staining of TUNEL and immunofluorescence labeling with different antibodies were performed and the positive cells were counted. In this study, we chose antiNeuN, anti-GFAP, and anti-CD31 antibodies, the markers of neuron, astrocyte, and vascular endothelial cell, combined with TUNEL staining to evaluate apoptosis and its location, and to further clarify the roles of multiple cells in NVU after ischemia and reperfusion.

At $24 \mathrm{~h}$ after reperfusion, rats were deep anesthetized and the brains were rapidly removed and cut into slices of $10 \mu \mathrm{m}$. We performed immunofluorescence labeling with anti-NeuN, anti-GFAP, and anti-CD31 antibodies and TUNEL staining with an In Situ Cell Death Detecting Kit
(Roche Diagnostics GmbH, Penzberg, Germany), respectively, according to the method described by Zhou et al. [42] and the manufacturer's protocol. Briefly, the brain slices were blocked with goat serum for $1 \mathrm{~h}$ and incubated with primary antibody at $4^{\circ} \mathrm{C}$ overnight. The slices were washed with PBS for $5 \mathrm{~min} \times 3$ and incubated with secondary antibodies for $60 \mathrm{~min}$ (light shielded) at $37^{\circ} \mathrm{C}$. Then the slices were stained with DAPI (Beyotime, Haimen, Jiangsu, china) for $15 \mathrm{~min}$. For TUNEL staining, the slices were incubated with TUNEL reaction mixture in a dark humidified chamber at $37^{\circ} \mathrm{C}$ for $1 \mathrm{~h}$, followed by a final wash for $10 \mathrm{~min} \times 3$ with PBS and then covered with glycerine. The captured images were viewed with a fluorescence microscope (Olympus/BX51, Tokyo, Japan). TUNEL positive cells and double stained cells of different groups were counted throughout five lesion regions randomly in penumbra of ischemic cortex.

2.10. Western Blot Analysis. Western blots were used to evaluate expression levels of proteins involved in PI3K/Akt pathway at $24 \mathrm{~h}$ after reperfusion. Whole cell protein of ischemic penumbra was extracted from the fresh brain. Equal volumes and qualities of protein solutions were separated by electrophoresis of appropriate concentrations of polyacrylamide gels and transferred to the poly-vinylidnene fluoride membranes (Millipore, Bedford, MA, USA). Then the membranes were placed in 5\% skim milk, prepared with Tris-buffered saline with $0.1 \%$ Tween-20 (TBST) to block nonspecific binding, and incubated with primary antibodies at $4^{\circ} \mathrm{C}$ overnight. In the following $2 \mathrm{~h}$, the membranes were incubated with horse-radish peroxidase-conjugated secondary anti-rabbit antibody after washing with TBST for $10 \mathrm{~min} \times 3$. Eventually, the targeted antigens were detected by standard chemical luminescence methods (Millipore, Bedford, MA, USA) with Fluor Chem FC 2 gel imaging system (Alpha Innotech, Santa Clara, CA, USA). GAPDH protein was used as a loading control. Phosphorylation levels of the targeted proteins were analyzed by total levels of corresponding proteins. Western blots were duplicated with three independent sets. Band intensities were measured with Quantity One software (Bio-Rad Laboratories, Hercules, CA, USA).

2.11. Statistical Analysis. When all the data have been collected, the animal grouping was released. Data are presented as the means \pm standard error of the mean (SEM). Statistical significance was determined by one-way ANOVA followed by Tukey's multiple comparison test or unpaired Student's $t$-tests using SPSS 11.5 for Windows (Chicago, IL, USA). All were considered statistically significant for $P$ values $<0.05$.

\section{Results}

3.1. Effects of XXMD on Cerebral Infarct Area and Neurological Deficits. The occlusion for $90 \mathrm{~min}$ followed by reperfusion for $24 \mathrm{~h}$ led to an infarct area and marked neurological deficits in rats. The results indicated that XXMD treatment resulted in a significant reduction in infarct area compared to the I/R group $(21.75 \pm 1.93 \%$ and $41.50 \pm 2.63 \%$, resp.). 
In addition, the rats subjected to cerebral ischemia and reperfusion showed noticeable neurological deficits which were markedly attenuated by XXMD treatment, while rats in the sham group performed normally after operation.

To evaluate the contribution of PI3K/Akt pathway to cerebral infarct area and neurological deficits, we investigated the effects of inhibiting PI3K with the specific inhibitor LY294002 on cerebral infarct area and neurological function at $24 \mathrm{~h}$ after reperfusion. Larger infarct area was observed in LY294002-treated rats $(39.01 \pm 2.27 \%)$ compared with the rats in the XXMD60 group. Additionally, we found a smaller infarct area in the XXMD60 + vehicle group (24.25 \pm $2.23 \%$ ) compared with the I/R group (Figures 2(a) and 2(b)). Likewise, LY294002 blocked the effects of XXMD60 on neurological deficits (Figure 2(c)). These findings were consistent with the hypothesis that XXMD-induced neuroprotection required the activation of PI3K/Akt pathway.

3.2. Effects of $X X M D$ on Neuronal Injury. Nissl staining showed neuronal injury in penumbra of ischemic cortex at $24 \mathrm{~h}$ after reperfusion. The images showed that most cells with intercellular space enlarged were shrunk in the ischemic cortex and had deep color staining indicative of injury in the I/R group. However, these characteristic changes were not observed in the sham group and were improved by XXMD treatment. Interestingly, LY294002 blocked the effect of XXMD on neuronal injury. Furthermore, there were more intact cells in the ischemic cortex of the XXMD-treated rats in the absence of LY294002 compared to the I/R group (Figure 3).

3.3. Effects of XXMD on Neuron Apoptosis. Double staining of TUNEL and immunofluorescence labeling with NeuN were performed to evaluate neuron apoptosis. As shown in Figure 4, XXMD strongly protected against neuron apoptosis in ischemic cortex at $24 \mathrm{~h}$ after reperfusion. The images of TUNEL staining revealed that $90 \mathrm{~min}$ of ischemia followed by reperfusion increased the number of TUNEL positive cells in ischemic cortex compared with the sham group, and XXMD markedly attenuated apoptosis, as indicated by a notable attenuation of the ischemia-induced increase in TUNEL positive cells. Additionally, the results of double staining showed that ischemia without XXMD treatment induced significant neuron apoptosis, whereas the number of TUNEL and NeuN double stained cells was markedly reduced in the XXMD60 group. However, PI3K inhibitor, LY294002, reduced neuron protection of XXMD, as indicated by a striking increase of neuron apoptosis. We further analyzed the percentage of apoptotic neurons in NeuN positive cells. The data indicated that there was a larger percentage of apoptotic cells in the I/R group. In striking contrast, we found that XXMD promoted neuronal survival, as indicated by a profound attenuation of the percentage of apoptotic neurons. However, the effects of XXMD were blocked by treatment with the PI3K inhibitor, LY294002.

3.4. Effects of XXMD on Astrocyte Apoptosis. To comprehensively determine apoptosis of different cells in NVU, we next performed double staining for TUNEL and immunofluorescence labeling with GFAP to evaluate astrocyte apoptosis. As represented in the ischemic cortex, the number of TUNEL and GFAP double stained cells was markedly increased in penumbra of ischemic cortex at $24 \mathrm{~h}$ after reperfusion. These changes were largely reversed in the XXMD60 group but worsened in the presence of LY294002. In all cases, double stained cells were hardly observed in the sham group. Percentages of apoptotic astrocyte in different groups were similar with the above findings (Figure 5).

The data revealed that astrocyte, as the key component of NVU, was involved in apoptosis induced by stroke with reperfusion. However, astrocyte apoptosis was decreased by treatment with XXMD. In view of the important role of astrocyte, the results further indicated that there were more normal astrocytes which promoted neuronal survival by XXMD treatment.

3.5. Effects of XXMD on Vascular Endothelial Cell Apoptosis. Intriguingly, the results of CD31 and TUNEL double staining in different groups were consistent with antiapoptotic effects of XXMD on neuron and astrocyte after ischemia and reperfusion. As reperfusion occurred, vascular endothelial cell, another considerable component of NVU, was subjected to severe damage unavoidably and showed the changes of apoptosis. However, XXMD treatment significantly reduced vascular endothelial cell apoptosis which was prevented by intracerebroventricular injection of LY294002 (Figure 6).

3.6. Effects of XXMD on the Expression of Proteins Involved in PI3K/Akt Pathway. Since the effects of the PI3K inhibitor indicated that XXMD may exert NVU protection via PI3K/Akt signaling pathway, we further examined whether XXMD regulated PI3K/Akt pathway following focal cerebral ischemia and reperfusion. The expression levels of related proteins in the penumbra of ischemic area were detected by Western blots. As shown in Figures 7 and 8, the results revealed that ischemia and reperfusion significantly induced remarkable decreases in the expression levels of p-PDK1, pAkt, and p-GSK3 $\beta$, whereas XXMD treatment maintained the phosphorylation levels of these proteins, which were reversed upon pretreatment with LY294002. Changes in total levels of PDK1, Akt, and GSK3 $\beta$ were not observed in different groups at $24 \mathrm{~h}$ after reperfusion. Additionally, our data indicated that p-PTEN levels were decreased after ischemia and reperfusion, and XXMD treatment attenuated the declines in p-PTEN after ischemia. However, LY294002 administration had no effect on the expression of p-PTEN in the XXMD-treated rats. Another surprising finding in this study was that XXMD treatment in the absence or presence of LY294002 effectively upregulated the expression of p-c-Raf, which was decreased after cerebral ischemia and reperfusion. Moreover, the data showed that total PTEN and c-Raf levels of different groups were not significantly different. 


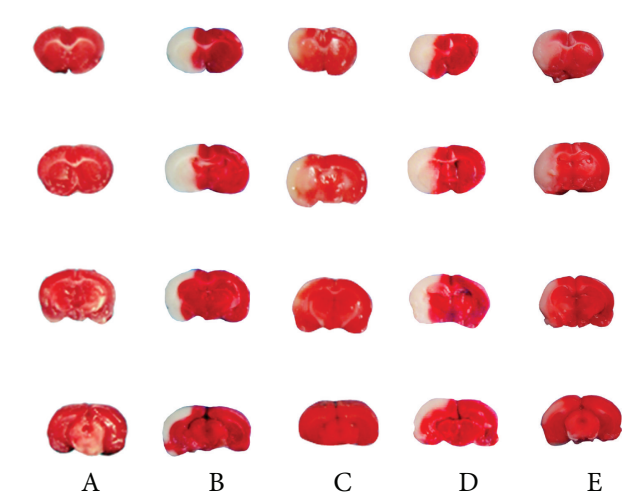

(a)

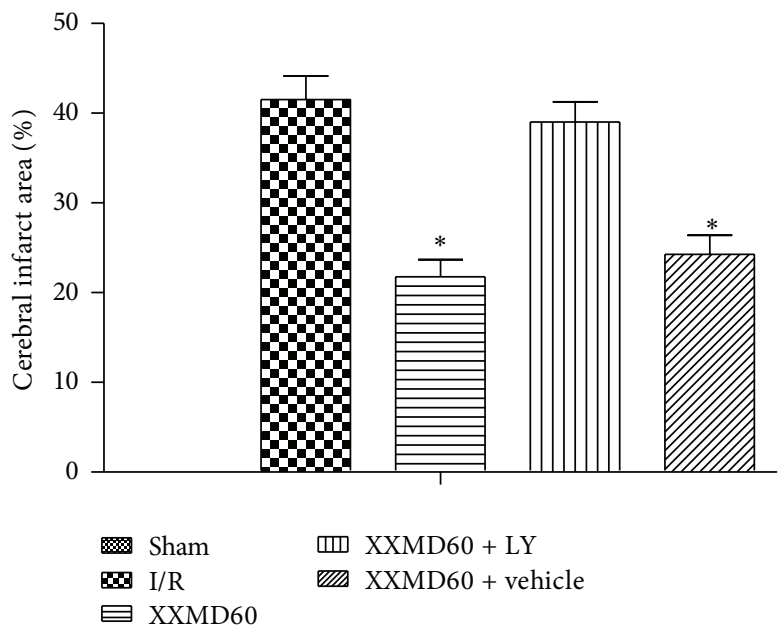

(b)

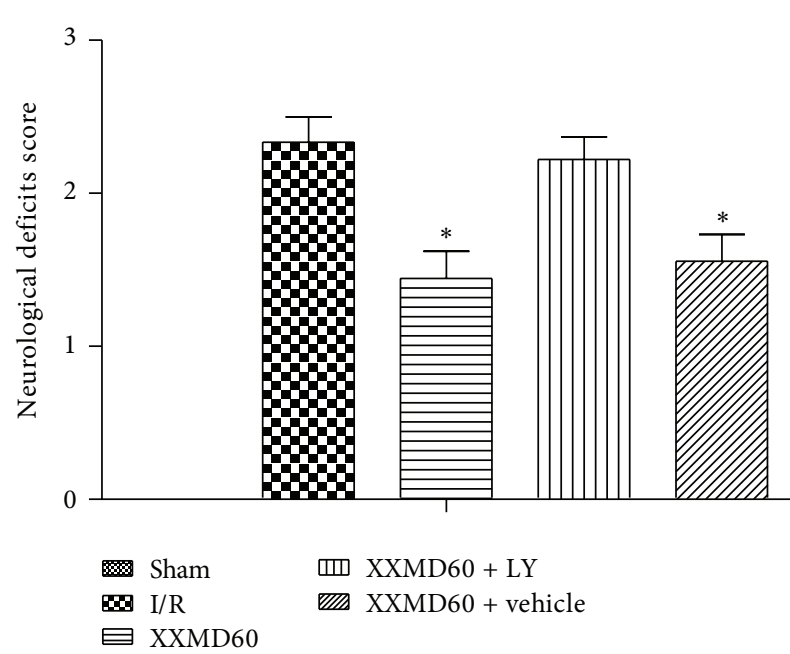

(c)

FIGURE 2: Cerebral infarct area and neurological deficits score at $24 \mathrm{~h}$ after reperfusion. (a) Representative images of brain slices stained with $1 \%$ TTC. The infarct area appeared white, whereas noninfarct areas were stained red purple. (b) Quantitative analysis of cerebral infarct areas. XXMD treatment reduced infarct area and LY294002 partially abolished the reduction in infarct area induced by XXMD treatment. (c) Effect of XXMD on neurological deficits score. XXMD treatment significantly improved neurological deficits score and LY294002 reversed effects of XXMD treatment. A, sham group; B, I/R group; C, XXMD60 group; D, XXMD60 + LY294002 group; E, XXMD60 + vehicle group; LY, LY294002. Data are reported as the means \pm SEM. $n=5 ;{ }^{*} P<0.05$ versus the I/R group.

\section{Discussion}

Stroke, as one of the leading causes of human death and disability, has become a severe threat to human health. Although the researchers have conducted plenty of preclinical studies and obtained many striking outcomes, translating bench success to the bedside proof of efficacy and safety has been frustrating [22]. There is a pressing need to resemble the human stroke with a satisfactory and appropriate animal model and scientifically evaluate the neuroprotective strategies [22]. It is well known that middle cerebral artery occlusion (MCAO) is one main cause of human ischemic strokes [43]. MCAO model, of which the employed procedures are noninvasive and easy to perform, could mimic the pathophysiological changes in human stroke and create reproducible territory infarct of MCA. Moreover, MCAO is non-expensive and enables the investigators to monitor the physiologic parameters during the experiments and analyze the outcome measures [38]. As much, MCAO has been one of the classic methods to induce the focal cerebral ischemia and reperfusion injury and test neuroprotective agents. In recent years, several scientists have focused on TCM, a potential and promising therapeutic approach for cerebral ischemia and investigated its efficacy and mechanism. XXMD is beneficial for the patients with stroke. A spate of studies has indicated that XXMD is prepared from twelve medical herbs containing a number of active fractions [44-46]. The effective components of XXMD such as paeoniflorin [47-49], baicalin [50-53], wogonin [54, 55], Baicalein [56-60], glycyrrhizic acid [61], and tetramethylpyrazine $[62,63]$ have been identified that they have antioxidant, anti-inflammatory, and antiapoptotic properties and could achieve neuroprotective effects following stroke with reperfusion. A better knowledge of the effects and underlying mechanisms of XXMD is very important to link the data from preclinical studies to clinical effects. Thus, we induced the focal cerebral ischemia 

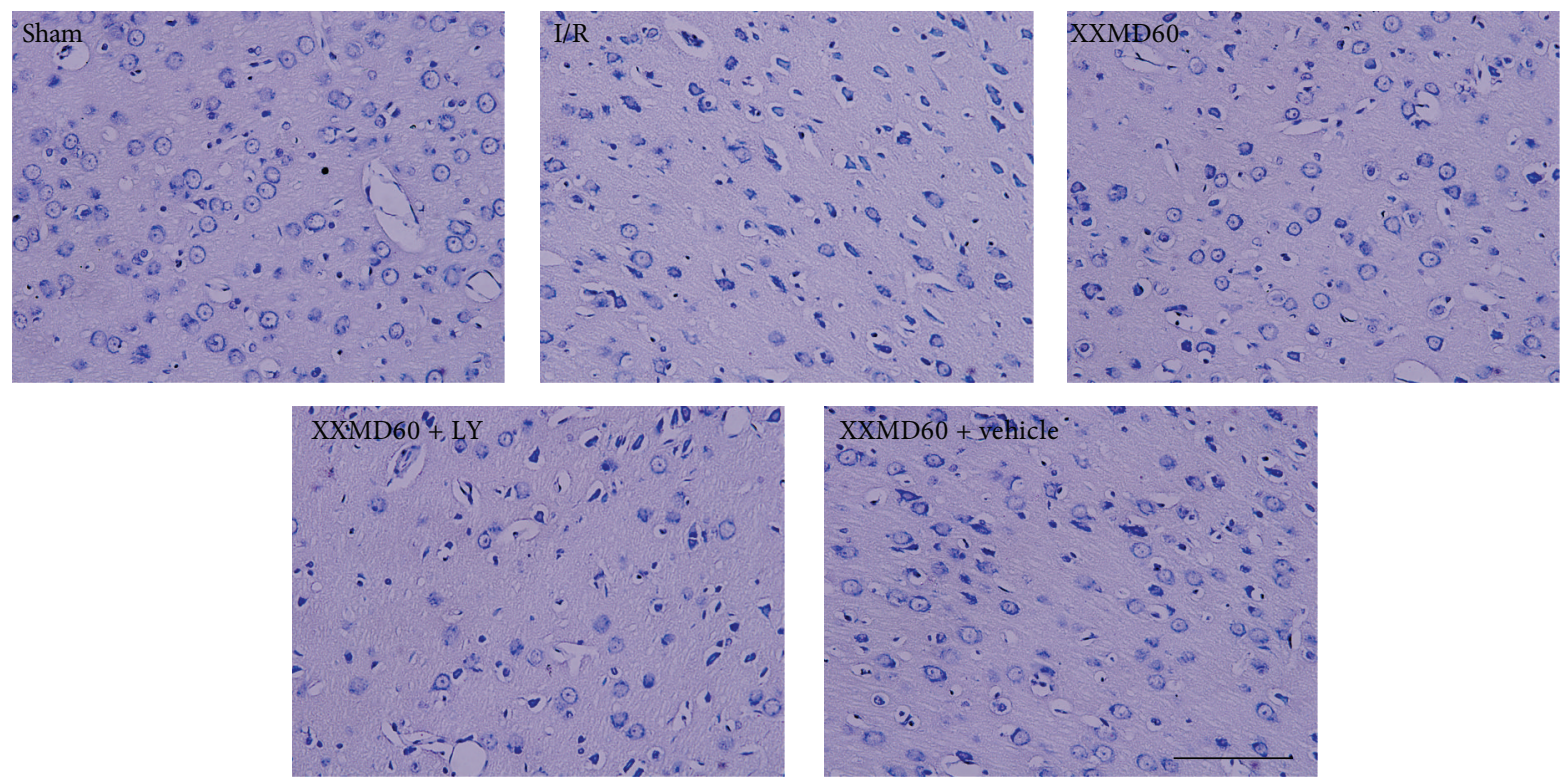

(a)

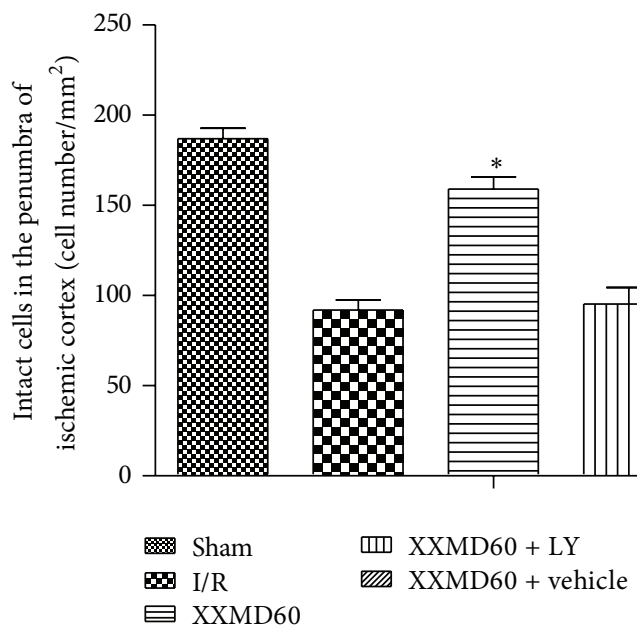

(b)

FIGURE 3: Representative images of Nissl staining and analysis of intact cells in different groups at $24 \mathrm{~h}$ after reperfusion. (a) The images of Nissl staining. Normal neurons were arranged orderly and had normal morphology with intact structure, abundant cytoplasm, and clear nucleolus in sham group. Most neurons in the damaged area appeared shrunken and deep stained in the I/R group. XXMD treatment improved the ischemic changes, which were blocked by LY294002. (b) Analysis of intact cells in penumbra of ischemic area. The number of intact cells in XXMD60 group was significantly higher than that in the I/R group. LY, LY294002. Scale bar $=50 \mu \mathrm{m}$. Data are reported as the means \pm SEM. $n=6 ;{ }^{*} P<0.05$ versus the I/R group.

and reperfusion injury with MCAO and selected the different doses of XXMD according to common human daily dose to observe the effects of XXMD on ischemic injury. Based on the preliminary results, we eventually determined the optimal dosages $(60 \mathrm{~g} / \mathrm{kg} / \mathrm{d})$ for the following exploration.

In the present study, we further investigated the NVU protective effects of XXMD after ischemia and reperfusion. The results clearly demonstrated that PI3K/Akt pathway played the crucial roles in NVU protection of XXMD against focal cerebral ischemia and reperfusion injury in rats.

Increased attention has recently been drawn to NVU protection which has quite significant implications for cerebral ischemia and reperfusion injury. Given to its important role, we focused on evaluating apoptosis of neuron, astrocyte, and vascular endothelial cell, respectively, which were important components of NVU. The results suggested that apoptosis was a common event after ischemia and reperfusion. Although apoptosis mainly located in neurons, other components of NVU were also involved in this event, such as astrocytes and vascular endothelial cells. These results further indicated that it was necessary to pay attention to NVU protection in treatment of stroke. XXMD had multiple targets to reduce apoptosis of different cells in NVU. Moreover, obvious improvements of neurological deficits and infarct 

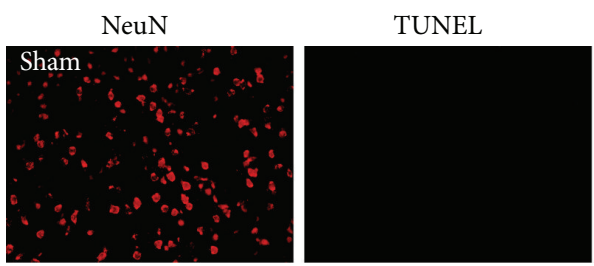

TUNEL + DAPI
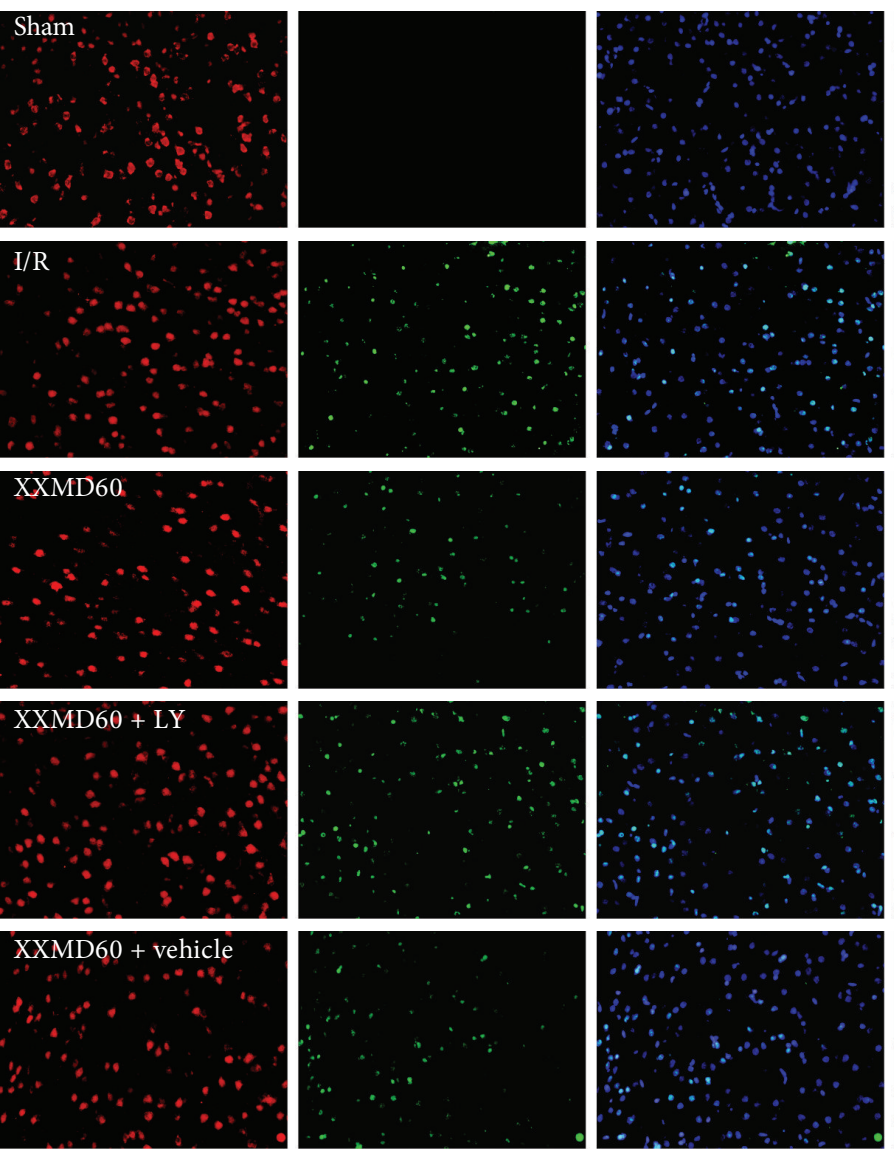

(a)
NeuN + TUNEL
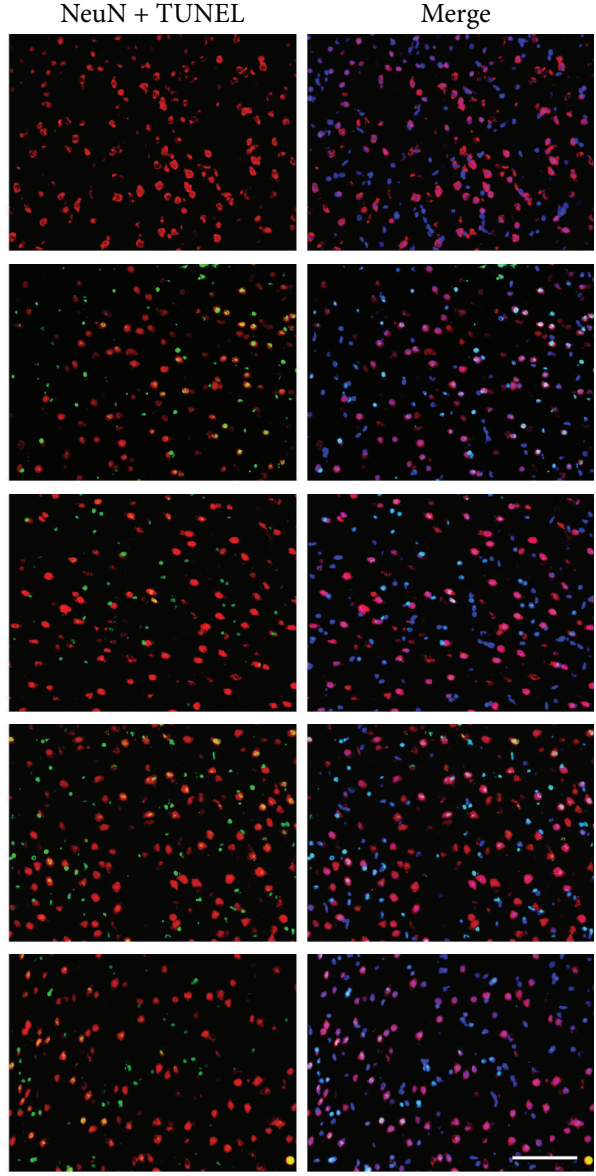

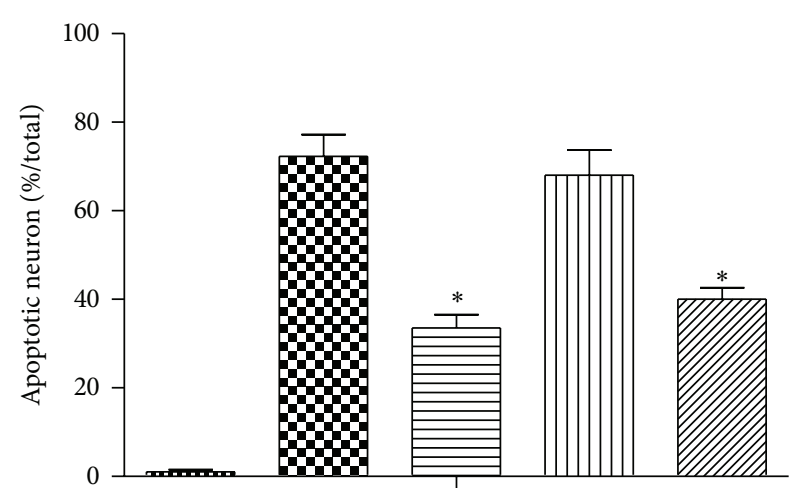
Sham
$\theta \mathrm{I} / \mathrm{R}$
띠 XXMD60 + LY

$$
\begin{aligned}
& \text { Sham } \\
& \text { I/R } \\
& \Xi \text { XXMD60 }
\end{aligned}
$$

WXM $\mathrm{XX0}+$ vehicle

(b)

FIGURE 4: Representative images of neuron apoptosis of different groups in penumbra of ischemic cortex at $24 \mathrm{~h}$ after reperfusion. (a) Representative photomicrographs of immunofluorescence labeling with NeuN (red) and TUNEL (green) double staining. Nuclei were counterstained with DAPI (blue), and collocation of green and blue indicated TUNEL positive cell. At $24 \mathrm{~h}$ after reperfusion, a significant number of TUNEL-positive cells were observed in ischemic cortex of the I/R group and were strongly attenuated by XXMD treatment. (b) Quantification of apoptotic neurons. TUNEL and NeuN double stained cells (yellow) indicated the apoptotic neurons which were significantly induced by reperfusion and decreased by XXMD treatment. (c) Percentage of apoptotic neuron in NeuN positive cell. Percentage of apoptotic neuron was increased after stroke with reperfusion and was markedly reduced by XXMD treatment. However, neuron apoptosis was worsened by treatment with LY294002. LY, LY294002. Scale bar $=50 \mu \mathrm{m}$. Data are reported as the means \pm SEM. $n=6 ;{ }^{*} P<0.05$ versus the I/R group. 

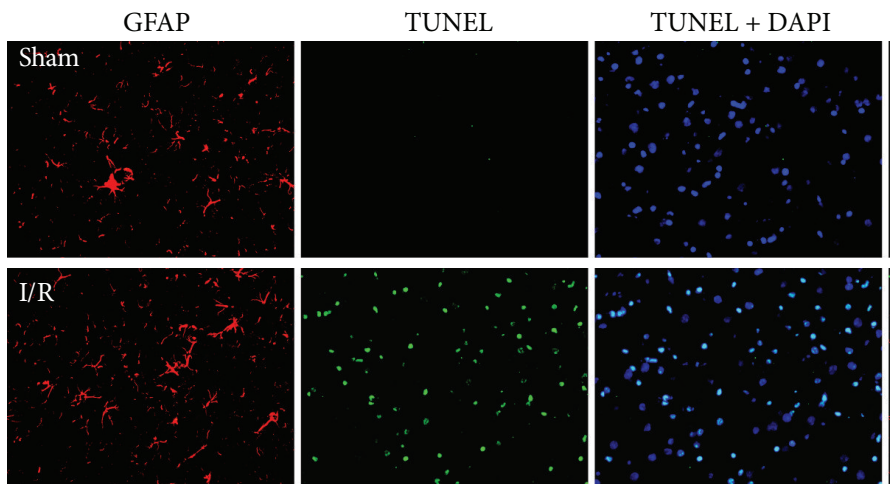

GFAP + TUNEL
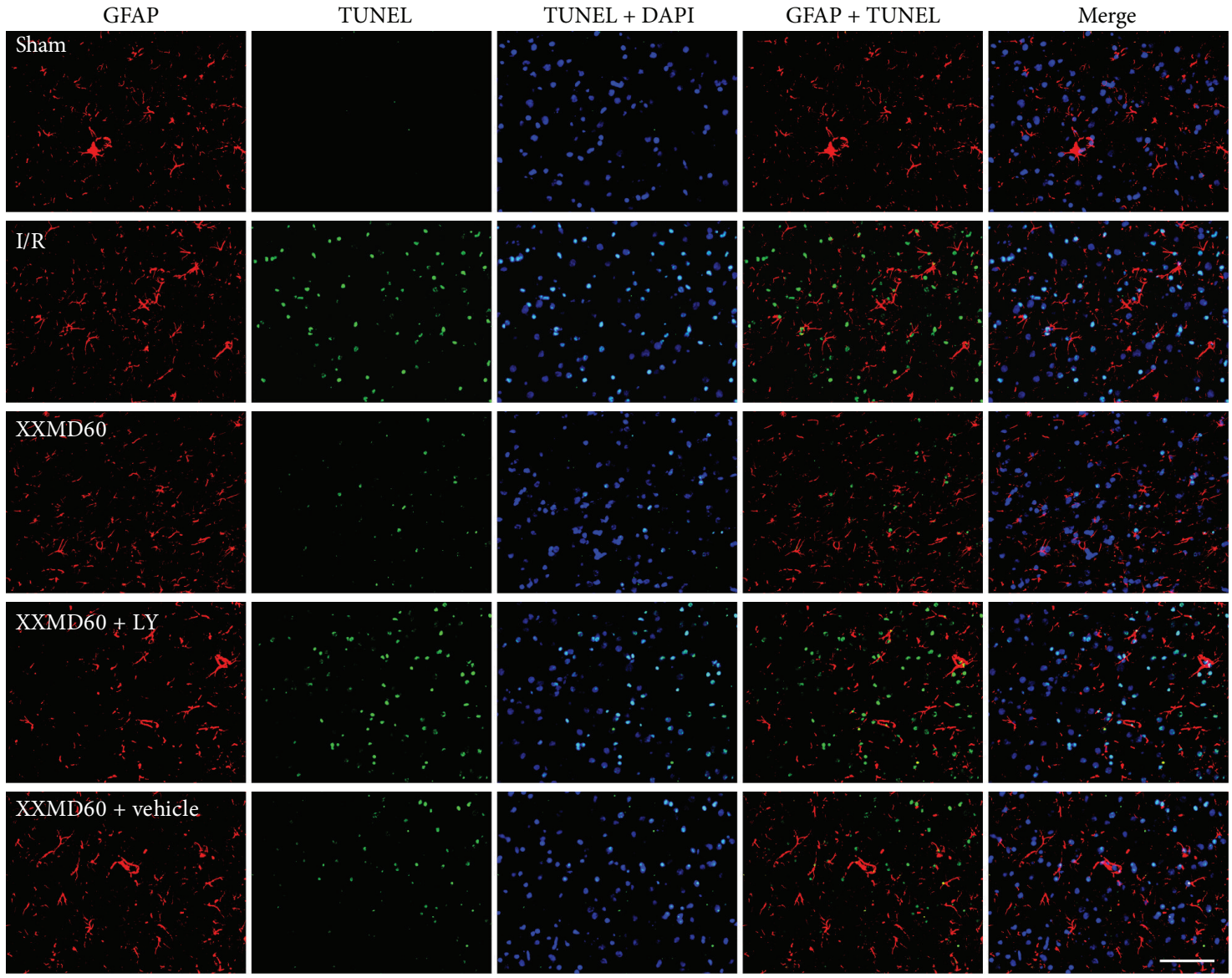

(a)

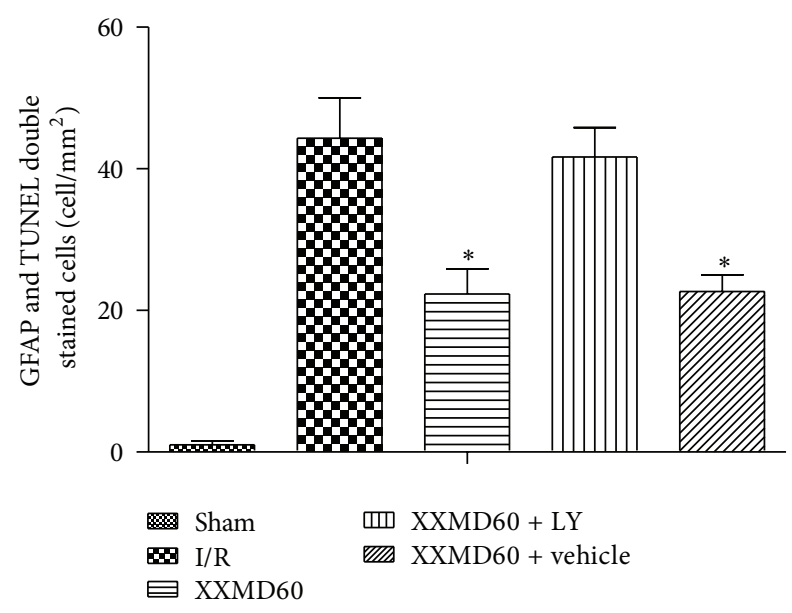

(b)

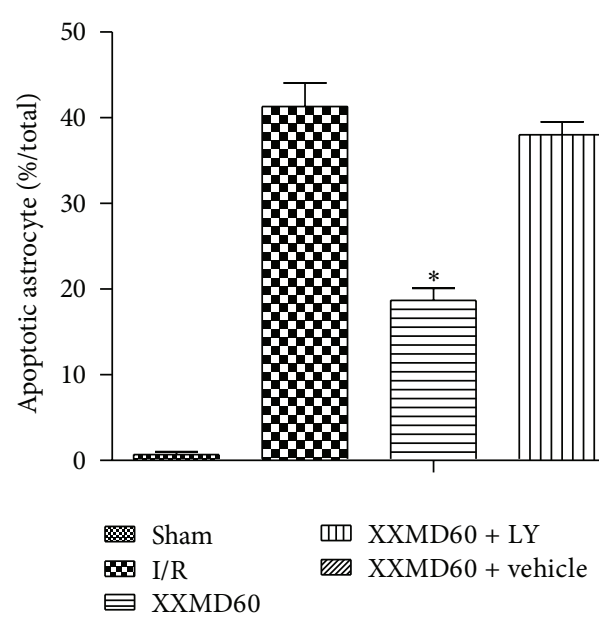

(c)

FIGURE 5: Representative images of astrocyte apoptosis in penumbra of ischemic cortex at $24 \mathrm{~h}$ after reperfusion. (a) Representative photomicrographs of immunofluorescence labeling with GFAP (red) and TUNEL (green) double staining. Nuclei were counterstained with DAPI (blue). And collocation of green and blue indicated TUNEL positive cell in the same view. Stroke with reperfusion notably caused TUNEL-positive cells at $24 \mathrm{~h}$ after reperfusion without XXMD treatment, and a decrease was found in XXMD60 group. However, LY294002 abolished the reduction. (b) Quantification of apoptotic astrocytes. TUNEL and GFAP double stained cells (yellow) indicated the apoptotic astrocytes. A marked increase in apoptotic astrocytes was found at $24 \mathrm{~h}$ after reperfusion and was reversed by XXMD treatment. (c) Percentage of apoptotic astrocyte in GFAP positive cell. There were more nonapoptotic astrocytes in the XXMD-treated groups in absence of LY294002 compared with the I/R group. LY, LY294002. Scale bar $=50 \mu \mathrm{m}$. Data are reported as the means \pm SEM. $n=6 ;{ }^{*} P<0.05$ versus the I/R group. 

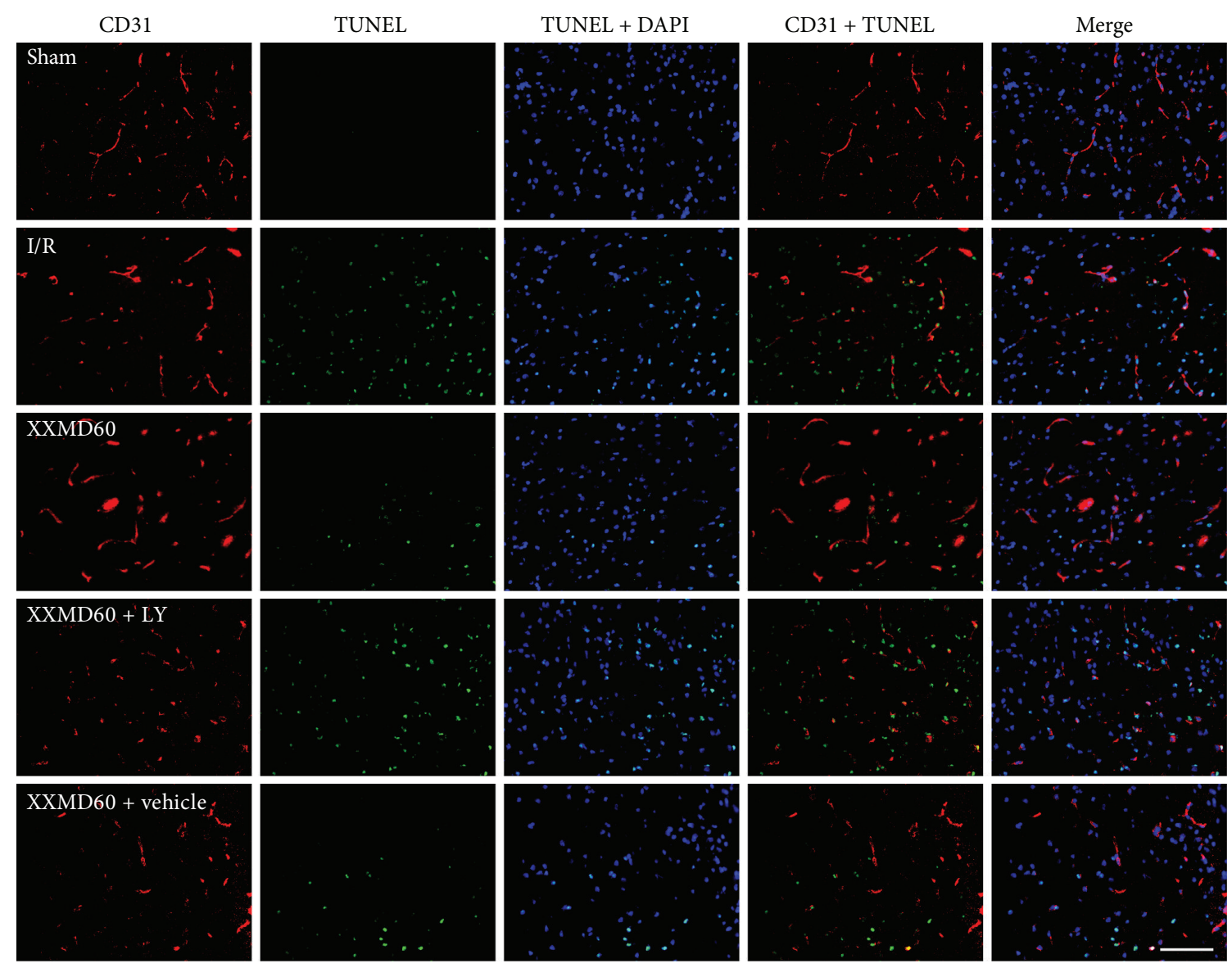

(a)

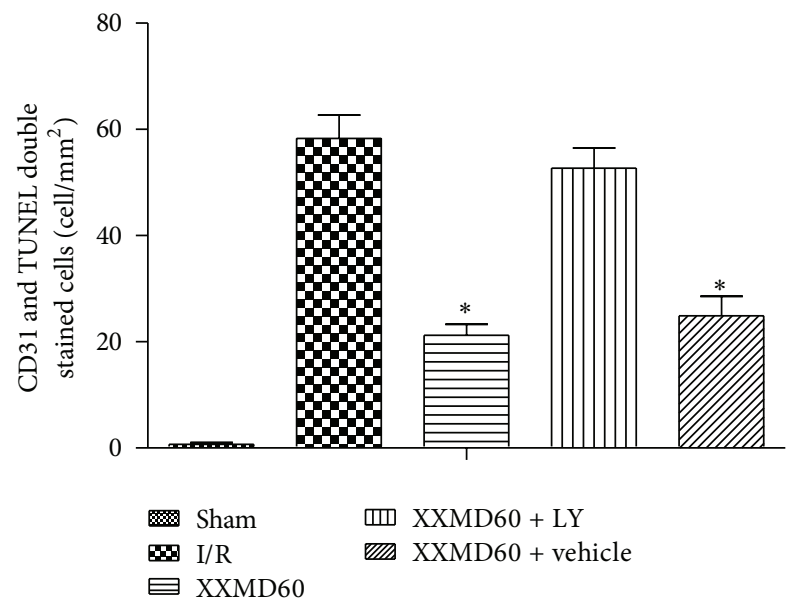

(b)

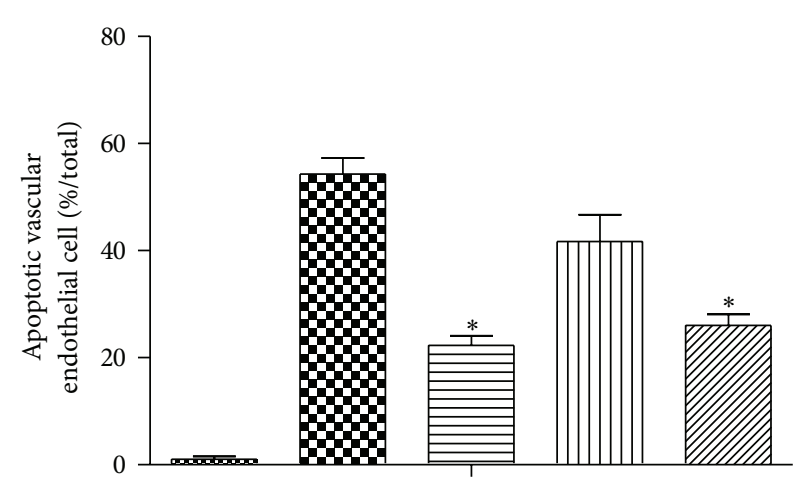
Sham
$8 \mathrm{I} / \mathrm{R}$
四 XXMD60 + LY
XXMD60 + vehicle

$\sqsupseteq$ XXMD60

(c)

FIGURE 6: Representative images of vascular endothelial cell apoptosis in penumbra of ischemic cortex at $24 \mathrm{~h}$ after reperfusion. (a) Representative photomicrographs of immunofluorescence labeling with CD31 (red) and TUNEL (green) double staining. Nuclei were counterstained with DAPI (blue). And collocation of green and blue indicated TUNEL positive cell in the same view. (b) Quantification of apoptotic vascular endothelial cells. TUNEL and CD31 double stained cells (yellow) indicated the apoptotic vascular endothelial cells. Cerebral ischemia and reperfusion induced a significant increase in apoptotic vascular endothelial cell which was prevented by XXMD treatment. However, LY294002 inhibited the antiapoptotic effect of XXMD on vascular endothelial cell. (c) Percentage of apoptotic vascular endothelial cell in CD31 positive cell. There were more nonapoptotic vascular endothelial cells in the XXMD-treated groups in the absence of LY294002 compared with the I/R group. LY, LY294002. Scale bar $=50 \mu \mathrm{m}$. Data are reported as the means \pm SEM. $n=6 ;{ }^{*} P<0.05$ versus the I/R group. 
p-PDK1 (Ser241)

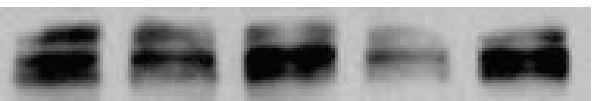

PDK1
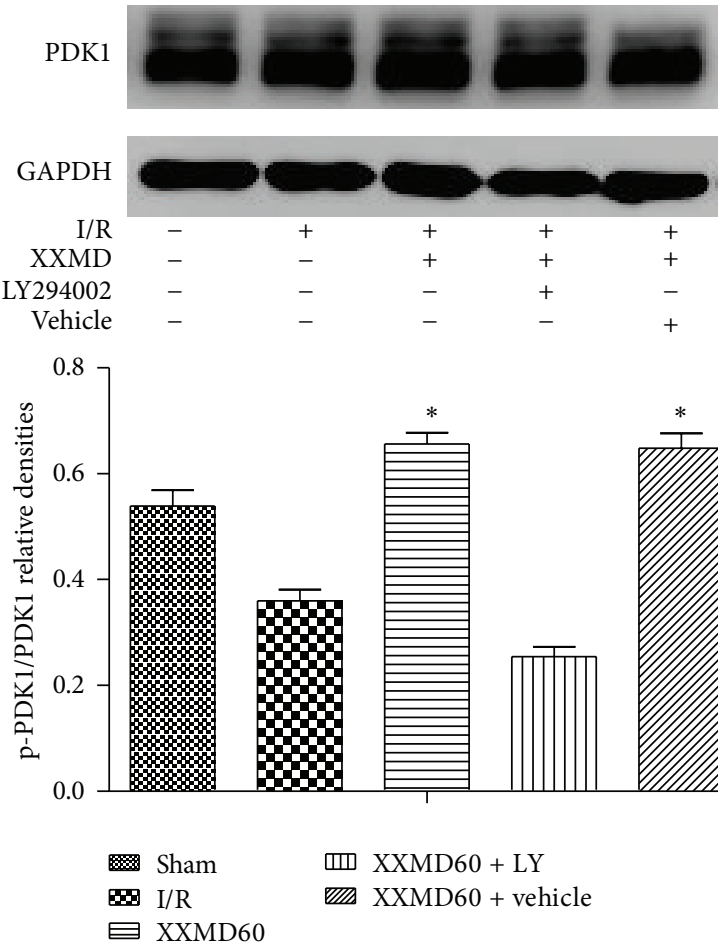

(a)
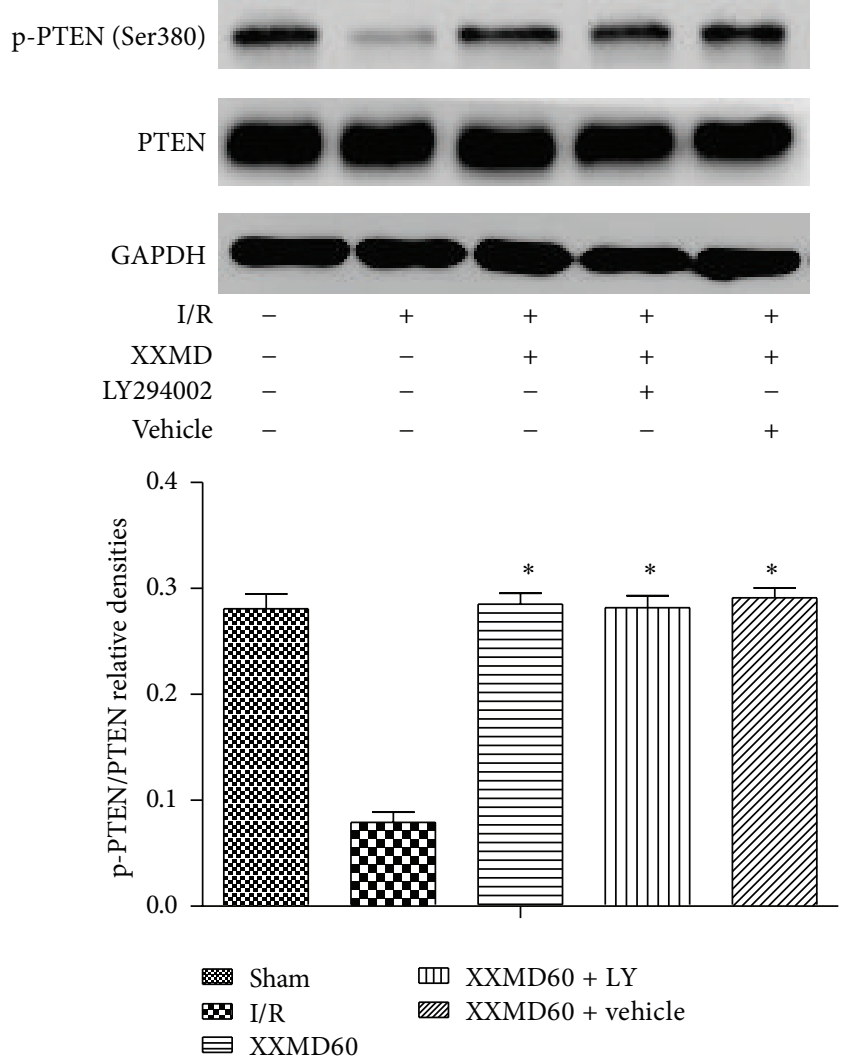

(b)

Figure 7: Western blot analysis of phosphorylation levels of PDK1, PTEN at $24 \mathrm{~h}$ after reperfusion. (a) Representative protein bands for p-PDK1 (Ser241), total PDK1, were shown along with their corresponding relatively densities. Although the levels of p-PDK1 (Ser241) were significantly decreased at $24 \mathrm{~h}$ after reperfusion, XXMD treatment blocked such changes. However, the effects were partly blocked by LY294002. No changes in PDK1 were detected in rats of different groups. GAPDH was used to show equal protein loading of each lane. (b) Representative protein bands for p-PTEN (Ser380), total PTEN. Relative densities demonstrated that p-PTEN (Ser380) expression levels were decreased at $24 \mathrm{~h}$ after ischemia. Levels of p-PTEN (Ser380) in the XXMD-treated rats were significantly higher than those in the I/R group, suggesting that XXMD treatment upregulated protein levels of p-PTEN (Ser380) after stroke with reperfusion and LY294002 had no effect on its expression. No changes in PTEN were observed in rats of different groups. Data are reported as the means \pm SEM. $n=5 ;{ }^{*} P<0.05$ versus the I/R group.

area were also observed in XXMD-treated group at $24 \mathrm{~h}$ after reperfusion.

How did XXMD exert its NVU protection against cerebral ischemia and reperfusion injury and which signaling pathway was involved in this course? These problems need to be explored. In this study, we stressed on PI3K/Akt pathway and identified the precise and underlying mechanisms. PI3K inhibitor, LY294002, was applied to determine the role of PI3K/Akt pathway in the study, such as analyses of cerebral infarct area, neurological function, and apoptosis. All findings suggested that PI3K was involved in the effect of XXMD on NVU protection. Otherwise, the results of Western blots which were next performed showed that phosphorylation levels of PTEN, PDK1, Akt, GSK3 $\beta$, and c-Raf were decreased in the penumbra of ischemic cortex at $24 \mathrm{~h}$ after reperfusion, and XXMD treatment improved phosphorylation levels of these proteins. The data also suggested that LY294002 reversed the effects of XXMD on phosphorylation levels of related proteins except for PTEN and c-Raf. These implicated that PI3K/Akt pathway contributed to neurovascular unit protection of XXMD after stroke with reperfusion (Figure 9).

Phosphoinositide dependent protein kinase-1 (PDK1) as the upstream of Akt may activate Akt by directly phosphorylating Thr308 and indirectly phosphorylating Ser473 [8, 64] and some research shows that ischemia and reperfusion induce a decrease of p-PDK1 [39]. In this study, we observed a reduction in $\mathrm{p}-\mathrm{PDK} 1$ and attenuation of that effect by XXMD treatment at $24 \mathrm{~h}$ after reperfusion, but this effect was blocked in the presence of LY294002. Zhao et al. [39] have reported that the levels of p-Akt and p-PDK1 are discordant after reperfusion, which is consistent with our study. It may be that p-Akt is autophosphorylated or phosphorylated by other kinases [65]. However, the concrete mechanism requires additional investigation.

Plenty of reports have shown that PTEN which prevents recruitment of Akt to the membrane for phosphorylation is a negative regulator of Akt $[64,66]$ and downregulation of PTEN expression attenuates brain damage induced by 

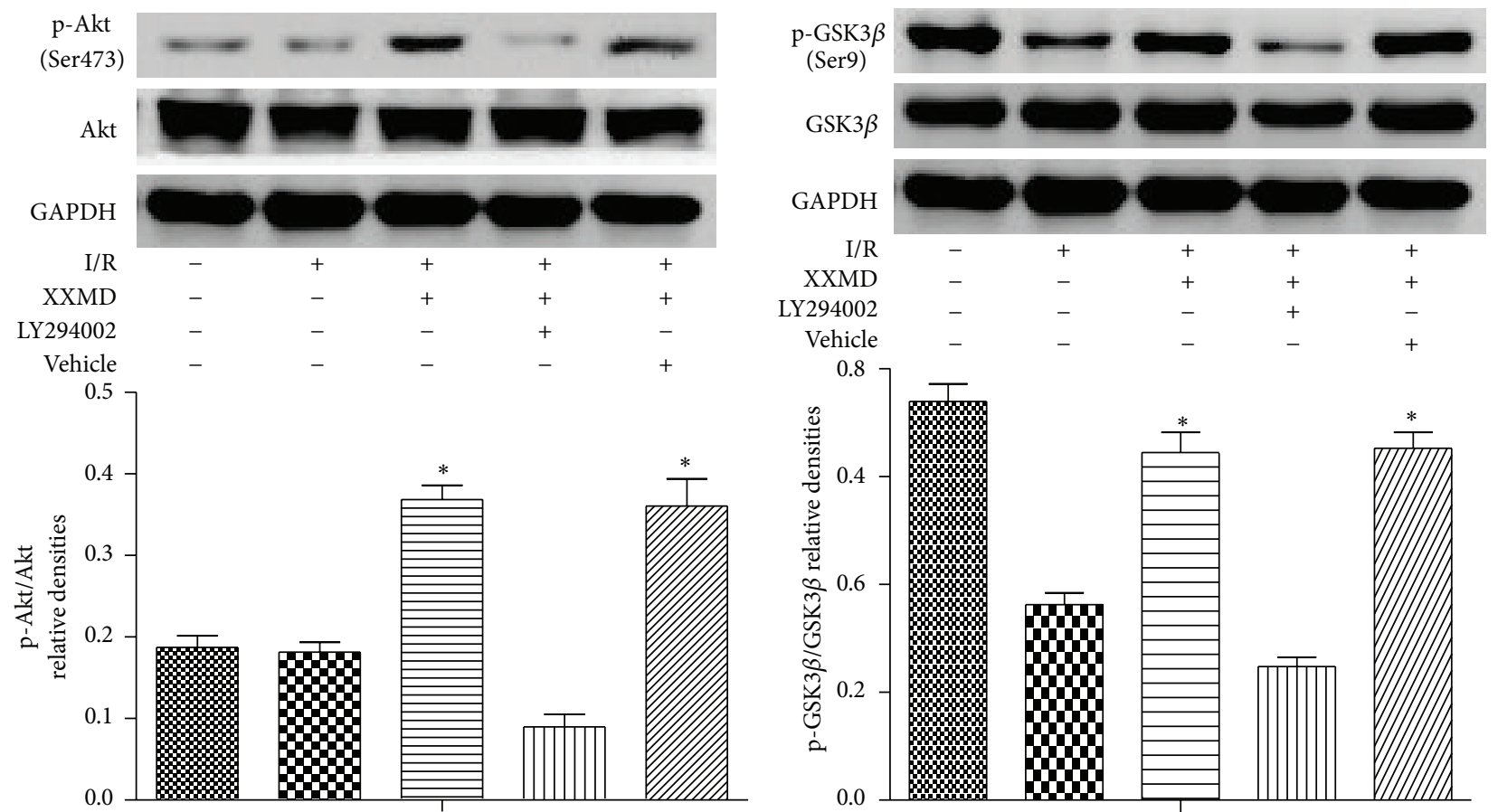

(a)

(b)

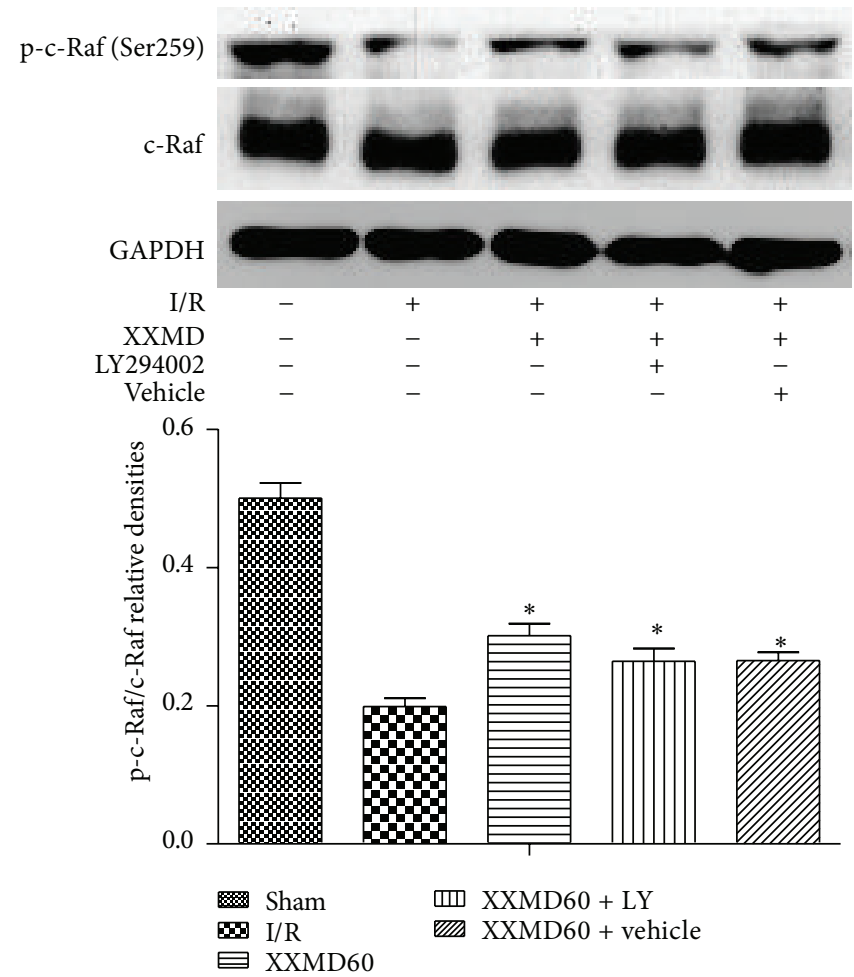

(c)

FIGURE 8: Western blot analysis of phosphorylation levels of Akt, GSK3 $\beta$, and c-Raf at $24 \mathrm{~h}$ after reperfusion. (a) Representative protein bands for p-Akt (Ser473), total Akt. p-Akt (Ser473) expression levels significantly were decreased at $24 \mathrm{~h}$ after reperfusion, whereas XXMD treatment enhanced p-Akt (Ser473) levels and the effects could be partly reversed by PI3K inhibitor. No changes in Akt were detected in rats of different groups. GAPDH was used to show equal protein loading of each lane. (b) Representative protein bands for p-GSK3 $\beta$ (Ser9), total GSK3 $\beta$. A decrease in p-GSK3 $\beta$ (Ser9) level was observed in the peripheral area of ischemia after reperfusion and the levels of p-GSK3 $\beta$ (Ser9) in the XXMD60 group were higher than those in the I/R group. However, inhibition of PI3K using LY294002 abolished the increase. No changes in GSK3 $\beta$ were observed in rats of different groups. (c) Representative protein bands for p-c-Raf, total c-Raf. Although p-c-Raf expression levels significantly were decreased at $24 \mathrm{~h}$ after reperfusion, XXMD preserved the levels of p-c-Raf. Notably, LY294002 did not significantly block the effect of XXMD on p-c-Raf. No changes in c-Raf were detected in rats of different groups. Data are reported as the means \pm SEM. $n=5 ;{ }^{*} P<0.05$ versus the I/R group. 


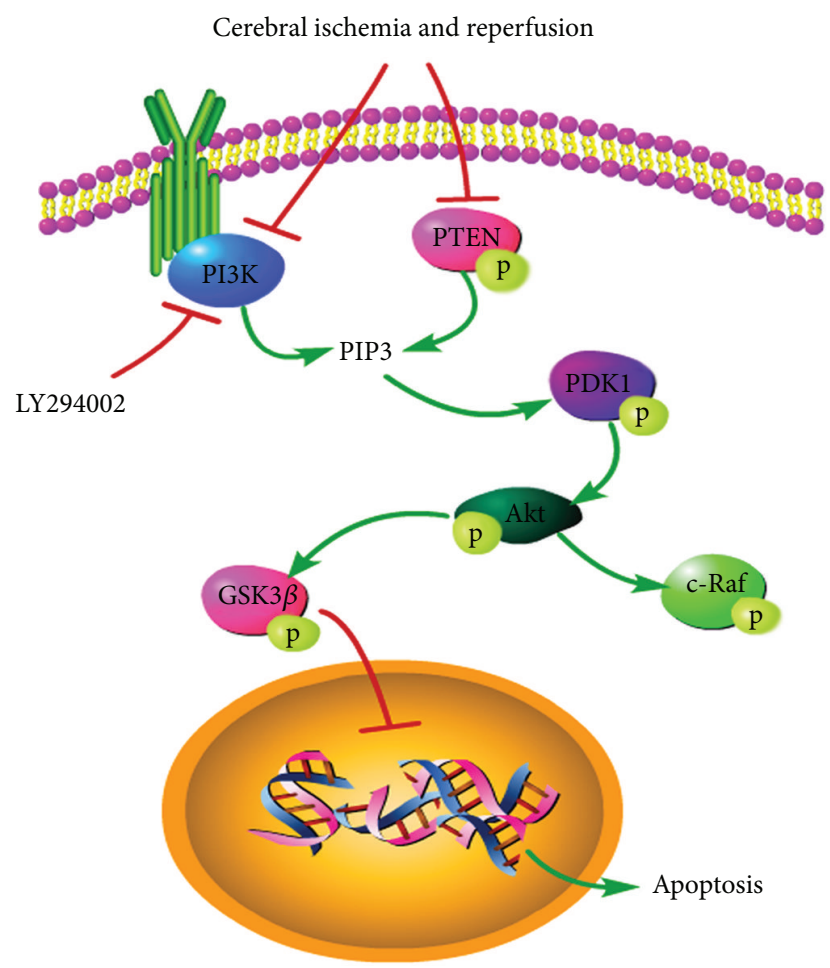

FIGURE 9: The diagram of the cell signaling pathway which was involved in NVU protection of XXMD against cerebral injury following focal cerebral ischemia and reperfusion. Reperfusion after stroke induced dysfunction of PI3K/Akt pathway and the PI3K/Akt inhibition led to dephosphorylation of PDK1, Akt, and GSK3 $\beta$ degradation, resulting in apoptosis. However, XXMD treatment inhibited apoptosis of different cells in NVU and increased expression levels of p-PTEN, p-PDK1, p-Akt, and p-GSK3 $\beta$. Furthermore, $\mathrm{PI} 3 \mathrm{~K} / \mathrm{Akt}$ pathway is associated with Ras/MAPK pathway. In the present study, we also found that XXMD upregulated the level of p-c-raf. Above all, all the observations in the study indicated that XXMD may exert its NVU protection partly through the activation of PI3K/Akt signaling pathway.

ischemia [67-69]. However, the role of p-PTEN in cerebral ischemia remains controversial. Lee et al. [70] have suggested that a decline of p-PTEN contributes to the reduction of ischemic damage. In the study, our findings showed that $\mathrm{p}$ PTEN was decreased at $24 \mathrm{~h}$ after reperfusion which was consistent with Zhao et al. [39] and a decline blocked by XXMD treatment. However, LY294002 did not affect the effect of XXMD on p-PTEN expression. In addition, the level of p-PTEN was coincident with p-PDK1 and p-Akt. Therefore, we proposed that increased p-PTEN expression promoted cell survival after ischemia and reperfusion.

Akt, the serine-threonine kinase, is a key molecule in PI3K/Akt pathway and controls survival and apoptosis $[71,72]$ and Akt inhibits apoptosis after being phosphorylated. Accumulating evidence has suggested that the expression level of p-Akt (Ser473) could be upregulated temporarily at the onset of focal cerebral ischemia and downregulated at $24 \mathrm{~h}$ after reperfusion, but the expression of Akt was not significantly changed [8]. In other words, Akt plays its roles through phosphorylation rather than regulating its protein expression. In the current study, the results of Western blots showed that p-Akt was notably decreased at $24 \mathrm{~h}$ after reperfusion, and XXMD treatment prevented that decline but did not affect the expression of Akt. LY294002 as the special inhibitor of PI3K had striking effects on XXMD treatment that it partly blocked p-Akt expression.

Activated Akt downregulates GSK3 $\beta$ activity by phosphorylating it at Ser9 and further promotes neuronal survival $[7,73,74]$. To test downstream of Akt signaling pathway, we evaluated GSK $3 \beta$ phosphorylation of groups subjected to different administrations. The data showed a reduction in $\mathrm{p}-\mathrm{GSK} 3 \beta$ at $24 \mathrm{~h}$ after reperfusion, but it was blocked by XXMD treatment. Additionally, PI3K inhibitor was involved in PI3K/Akt pathway and partly prohibited the expression of $\mathrm{p}-\mathrm{GSK} 3 \beta$ in rats administrated with XXMD treatment.

c-Raf, a mitogen-activated protein kinase, is a crucial target of RAS/MARK pathway. Previous studies show that Akt mediates phosphorylation of c-Raf at Ser259 and inhibits Raf kinase activity [75], thus promotes cell survival. In the current study, we also found that there was a decline of $\mathrm{p}$ c-Raf expression which was reversed by XXMD treatment, but LY294002 did not change the level of p-c-Raf in XXMDtreated group. Whether RAS/MARK pathway is involved in the NUV protection of XXMD deserves further study.

Above all, we highlighted NVU protection of XXMD and elucidated the role of PI3K/Akt pathway during this course in the current study. The findings indicated that XXMD reduced cerebral infarct area and improved neurological function after ischemia and reperfusion. More importantly, we found that XXMD could protect NVU from ischemiainduced apoptosis and may exert its effects, at least in part, via the PI3K/Akt signaling pathway. Whether other pathways or mechanisms were involved in the effects of XXMD on cerebral ischemia and reperfusion injury needs our further exploration.

\section{Conclusion}

Focal cerebral ischemia and reperfusion injury were successfully induced by MCAO. Cellular apoptosis in NVU was increased in penumbra of ischemic cortex, and PI3K/Akt pathway dysfunction occurred after cerebral ischemia and reperfusion. The results indicated that treatment with XXMD reduced infarct area and cellular apoptosis and improved function outcomes after stroke. Moreover, we demonstrated that such protection may involve blunting the decreases in phosphorylation of PTEN, PDK1, Akt, GSK3 $\beta$, and c-Raf. Taken as a whole, these effects on PI3K/Akt pathway may help explain the NVU protection of XXMD. In light of these observations, our data revealed the underlying mechanisms of XXMD treatment for stroke and provided more support for clinical applications of XXMD.

\section{Authors' Contribution}

R. Lan and J. Xiang contributed equally to this work. 


\section{Acknowledgments}

This work was supported by the National Natural Science Foundation of China (Grant no. 81173389 and no. 81202813). The authors would like to thank all the member of their department for the experimental supports.

\section{References}

[1] E. H. Lo, T. Dalkara, and M. A. Moskowitz, "Mechanisms, challenges and opportunities in stroke," Nature Reviews Neuroscience, vol. 4, no. 5, pp. 399-415, 2003.

[2] R. L. Vangilder, C. L. Rosen, T. L. Barr, and J. D. Huber, "Targeting the neurovascular unit for treatment of neurological disorders," Pharmacology and Therapeutics, vol. 130, no. 3, pp. 239-247, 2011.

[3] R. Liu, T. T. Zhang, C. X. Wu, X. Lan, and G. H. Du, “Targeting the neurovascular unit: development of a new model and consideration for novel strategy for Alzheimer's disease," Brain Research Bulletin, vol. 86, no. 1-2, pp. 13-21, 2011.

[4] B. R. S. Broughton, D. C. Reutens, and C. G. Sobey, "Apoptotic mechanisms after cerebral ischemia," Stroke, vol. 40, no. 5, pp. e331-e339, 2009.

[5] S. T. Hou and J. P. MacManus, "Molecular mechanisms of cerebral ischemia-induced neuronal death," International Review of Cytology, vol. 221, pp. 93-148, 2002.

[6] Y. M. Zhu, C. C. Wang, L. Chen et al., "Both PI3K/Akt and ERK1/2 pathways participate in the protection by dexmedetomidine against transient focal cerebral ischemia/reperfusion injury in rats," Brain Research, vol. 1494, pp. 1-8, 2013.

[7] H. Endo, C. Nito, H. Kamada, T. Nishi, and P. H. Chan, "Activation of the Akt/GSK3 $\beta$ signaling pathway mediates survival of vulnerable hippocampal neurons after transient global cerebral ischemia in rats," Journal of Cerebral Blood Flow and Metabolism, vol. 26, no. 12, pp. 1479-1489, 2006.

[8] H. Zhao, R. M. Sapolsky, and G. K. Steinberg, "Phosphoinositide-3-kinase/Akt survival signal pathways are implicated in neuronal survival after stroke," Molecular Neurobiology, vol. 34, no. 3, pp. 249-269, 2006.

[9] A. Saito, T. Hayashi, S. Okuno, M. Ferrand-Drake, and P. H. Chan, "Overexpression of copper/zinc superoxide dismutase in transgenic mice protects against neuronal cell death after transient focal ischemia by blocking activation of the bad cell death signaling pathway," Journal of Neuroscience, vol. 23, no. 5, pp. 1710-1718, 2003.

[10] T. Kawano, M. Morioka, S. Yano et al., "Decreased Akt activity is associated with activation of forkhead transcription factor after transient forebrain ischemia in gerbil hippocampus," Journal of Cerebral Blood Flow and Metabolism, vol. 22, no. 8, pp. 926-934, 2002.

[11] J. Zhang, Z. Deng, J. Liao et al., "Leptin attenuates cerebral ischemia injury through the promotion of energy metabolism via the PI(3)K/Akt pathway," Journal of Cerebral Blood Flow \& Metabolism, vol. 33, no. 4, pp. 567-574, 2013.

[12] G. B. Mackensen, M. Patel, H. Sheng et al., "Neuroprotection from delayed postischemic administration of a metalloporphyrin catalytic antioxidant," Journal of Neuroscience, vol. 21, no. 13, pp. 4582-4592, 2001.

[13] B. Xing, H. Chen, M. Zhang et al., "Ischemic postconditioning inhibits apoptosis after focal cerebral ischemia/reperfusion injury in the rat," Stroke, vol. 39, no. 8, pp. 2362-2369, 2008.
[14] S. Y. Li, D. Yang, Z. J. Fu, T. Woo, D. Wong, and A. C. Lo, "Lutein enhances survival and reduces neuronal damage in a mouse model of ischemic stroke," Neurobiology of Disease, vol. 45, no. 1, pp. 624-632, 2012.

[15] S. S. Baliga, K. M. Jaques-Robinson, N. M. Hadzimichalis, R. Golfetti, and G. F. Merrill, "Acetaminophen reduces mitochondrial dysfunction during early cerebral postischemic reperfusion in rats," Brain Research, vol. 1319, pp. 142-154, 2010.

[16] M. Gelderblom, F. Leypoldt, J. Lewerenz et al., "The flavonoid fisetin attenuates postischemic immune cell infiltration, activation and infarct size after transient cerebral middle artery occlusion in mice," Journal Cerebral Blood Flow \& Metabolism, vol. 32, no. 5, pp. 835-843, 2012.

[17] R. L. Zhang, M. Chopp, N. Jiang et al., "Anti-intercellular adhesion molecule-1 antibody reduces ischemic cell damage after transient but not permanent middle cerebral artery occlusion in the Wistar rat," Stroke, vol. 26, no. 8, pp. 1438-1443, 1995.

[18] S. Kawasaki-Yatsugi, C. Ichiki, S. I. Yatsugi et al., "Neuroprotective effects of an AMPA receptor antagonist YM872 in a rat transient middle cerebral artery occlusion model," Neuropharmacology, vol. 39, no. 2, pp. 211-217, 2000.

[19] T. Baba, M. Kameda, T. Yasuhara et al., "Electrical stimulation of the cerebral cortex exerts antiapoptotic, angiogenic, and antiinflammatory effects in ischemic stroke rats through phosphoinositide 3-kinase/akt signaling pathway," Stroke, vol. 40, no. 11, pp. e598-e605, 2009.

[20] C. Berthet, H. Lei, J. Thevenet, R. Gruetter, P. J. Magistretti, and L. Hirt, "Neuroprotective role of lactate after cerebral ischemia," Journal of Cerebral Blood Flow and Metabolism, vol. 29, no. 11, pp. 1780-1789, 2009.

[21] J. Ros, N. Pecinska, B. Alessandri, H. Landolt, and M. Fillenz, "Lactate reduces glutamate-induced neurotoxicity in rat cortex," Journal of Neuroscience Research, vol. 66, no. 5, pp. 790-794, 2001.

[22] Y. D. Cheng, L. Al-Khoury, and J. A. Zivin, "Neuroprotection for ischemic stroke: two decades of success and failure," The Journal of The American Society for Experimental NeuroTherapeutics, vol. 1, no. 1, pp. 36-45, 2004.

[23] P. Huang, C. M. Zhou, Qin-Hu et al., "Cerebralcare Granule(R) attenuates blood-brain barrier disruption after middle cerebral artery occlusion in rats," Experimental Neurology, vol. 237, no. 2, pp. 453-463, 2012.

[24] J. Xiang, Y. P. Tang, P. Wu, J. P. Gao, and D. F. Cai, “Chinese medicine Nao-Shuan-Tong attenuates cerebral ischemic injury by inhibiting apoptosis in a rat model of stroke," Journal of Ethnopharmacology, vol. 131, no. 1, pp. 174-181, 2010.

[25] H. W. Wang, K. T. Liou, Y. H. Wang et al., "Deciphering the neuroprotective mechanisms of Bu-yang Huan-wu decoction by an integrative neurofunctional and genomic approach in ischemic stroke mice," Journal of Ethnopharmacology, vol. 138, no. 1, pp. 22-33, 2011.

[26] X. M. Li, X. C. Bai, L. N. Qin, H. Huang, Z. J. Xiao, and T. M. Gao, "Neuroprotective effects of Buyang Huanwu Decoction on neuronal injury in hippocampus after transient forebrain ischemia in rats," Neuroscience Letters, vol. 346, no. 1-2, pp. 2932, 2003

[27] J. J. Lee, W. H. Hsu, T. L. Yen et al., “Traditional Chinese medicine, Xue-Fu-Zhu-Yu decoction, potentiates tissue plasminogen activator against thromboembolic stroke in rats," Journal of Ethnopharmacology, vol. 134, no. 3, pp. 824-830, 2011.

[28] X. H. Zhu, S. J. Li, H. H. Hu, L. R. Sun, M. Das, and T. M. Gao, "Neuroprotective effects of Xiao-Xu-Ming decoction 
against ischemic neuronal injury in vivo and in vitro," Journal of Ethnopharmacology, vol. 127, no. 1, pp. 38-46, 2010.

[29] Y. H. Wang, X. L. He, H. G. Yang, H. L. Qin, and G. H. Du, "Effects of the effective components groups of Xiao-Xu-Ming decoction on MCAO rats," Chinese Pharmaceutical Journal, vol. 47, no. 3, pp. 94-198, 2012 (Chinese).

[30] Y. H. Wang, X. L. He, X. X. Lee, H. L. Qin, and G. H. Du, "Effects of the effective component group of Chinese herbal medicine Xiao-Xu-Ming decoction on brain mitochondria in rats with chronic cerebral ischemia," Journal of Chinese Integrative Medicine, vol. 10, no. 5, pp. 569-576, 2012 (Chinese).

[31] D. F. Cai, Y. K. Yang, X. X. Gu et al., "Clinical trial on treatment of acute cerebral infarction with TCM treatment according to syndrome differentiation combining Western medicine by staging," Zhongguo Zhong xi yi jie he za zhi Zhongguo Zhongxiyi jiehe zazhi $=$ Chinese journal of integrated traditional and Western medicine / Zhongguo Zhong xi yi jie he xue hui, Zhongguo Zhong yi yan jiu yuan zhu ban, vol. 27, no. 9, pp. 789792, 2007 (Chinese).

[32] S. Zhou, "Clinical Research on Xiao-Xu-Ming decoction treating the stroke patients with neurological deficits," Journal of New Chinese Medicine, vol. 43, no. 5, pp. 17-18, 2011 (Chinese).

[33] J. H. Huang, X. H. Huang, Z. Y. Chen, Q. S. Zheng, and R. Y. Sun, "Dose conversion among different animals and healthy volunteers in pharmacological study," Chinese Journal of Clinical Pharmacology and Therapeutics, vol. 9, no. 9, pp. 1069-1072, 2004 (Chinese).

[34] J. Xiang, R. Lan, Y. P. Tang, Y. P. Chen, and D. F. Cai, "Apocynum venetum leaf extract attenuates disruption of the blood-brain barrier and upregulation of matrix metalloproteinase-9/-2 in a rat model of cerebral ischemia-reperfusion injury," Neurochemical Research, vol. 37, no. 8, pp. 1820-1828, 2012.

[35] G. J. Zarow, H. Karibe, B. A. States, S. H. Graham, and P. R. Weinstein, "Endovascular suture occlusion of the middle cerebral artery in rats: effect of suture insertion distance on cerebral blood flow, infarct distribution and infarct volume," Neurological Research, vol. 19, no. 4, pp. 409-416, 1997.

[36] R. Schmid-Elsaesser, S. Zausinger, E. Hungerhuber, A. Baethmann, and H. J. Reulen, "A critical reevaluation of the intraluminal thread model of focal cerebral ischemia: evidence of inadvertent premature reperfusion and subarachnoid hemorrhage in rats by laser-Doppler flowmetry," Stroke, vol. 29, no. 10, pp. 2162-2170, 1998.

[37] Q. Zhao, H. Memezawa, M. L. Smith, and B. K. Siesjo, "Hyperthermia complicates middle cerebral artery occlusion induced by an intraluminal filament," Brain Research, vol. 649, no. 1-2, pp. 253-259, 1994.

[38] K. M. Sicard and M. Fisher, "Animal models of focal brain ischemia," Experimental \& Translational Stroke Medicine, vol. 1, article 7, 2009.

[39] H. Zhao, T. Shimohata, J. Q. Wang et al., "Akt contributes to neuroprotection by hypothermia against cerebral ischemia in rats," Journal of Neuroscience, vol. 25, no. 42, pp. 9794-9806, 2005.

[40] G. Paxinos and C. Watson, The Rat Brain in Stereotaxic Coordinates, Compact, San Diego Academic Press, California, Calif, USA, 3rd edition, 1997.

[41] H. Hara, P. L. Huang, N. Panahian, M. C. Fishman, and M. A. Moskowitz, "Reduced brain edema and infarction volume in mice lacking the neuronal isoform of nitric oxide synthase after transient MCA occlusion," Journal of Cerebral Blood Flow and Metabolism, vol. 16, no. 4, pp. 605-611, 1996.
[42] C. Zhou, J. Tu, Q. Zhang et al., "Delayed ischemic postconditioning protects hippocampal CA1 neurons by preserving mitochondrial integrity via Akt/GSK3beta signaling," Neurochemistry International, vol. 59, no. 6, pp. 749-758, 2011.

[43] G. J. Del Zoppo, K. Poeck, M. S. Pessin et al., "Recombinant tissue plasminogen activator in acute thrombotic and embolic stroke," Annals of Neurology, vol. 32, no. 1, pp. 78-86, 1992.

[44] Z. Li, K. Ni, and G. Du, "Simultaneous analysis of six effective components in the anti-Alzheimer's disease effective component group of Xiao-Xu-Ming decoction," Chinese Journal of Chromatography (Se Pu), vol. 25, no. 1, pp. 80-83, 2007 (Chinese).

[45] K. Du, C. Wu, C. Ding, S. Zhao, H. Qin, and J. Zhang, "Simultaneous LC-MS analysis and of wogonin and oroxylin a in rat plasma, and pharmacokinetic studies after administration of the active fraction from Xiao-Xu-Ming decoction," Chromatographia, vol. 69, no. 11-12, pp. 1259-1266, 2009.

[46] Y. Wang, C. Ding, K. Du et al., "Identification of active compounds and their metabolites by high-performance liquid chromatography/electrospray ionization Fourier transform ion cyclotron resonance mass spectrometry from Xiao-Xu-Ming decoction (XXMD)," Rapid Communications in Mass Spectrometry, vol. 23, no. 17, pp. 2724-2732, 2009.

[47] N. Y. Tang, C. H. Liu, C. T. Hsieh, and C. L. Hsieh, “The anti-inflammatory effect of paeoniflorin on cerebral infarction induced by ischemia-reperfusion injury in sprague-dawley rats," American Journal of Chinese Medicine, vol. 38, no. 1, pp. 51-64, 2010.

[48] D. M. Chen, L. Xiao, X. Cai, R. Zeng, and X. Z. Zhu, "Involvement of multitargets in paeoniflorin-induced preconditioning," Journal of Pharmacology and Experimental Therapeutics, vol. 319, no. 1, pp. 165-180, 2006.

[49] L. Xiao, Y. Z. Wang, J. Liu, X. T. Luo, Y. Ye, and X. Z. Zhu, "Effects of paeoniflorin on the cerebral infarction, behavioral and cognitive impairments at the chronic stage of transient middle cerebral artery occlusion in rats," Life Sciences, vol. 78, no. 4, pp. 413-420, 2005.

[50] X. K. Tu, W. Z. Yang, R. S. Liang et al., "Effect of baicalin on matrix metalloproteinase-9 expression and blood-brain barrier permeability following focal cerebral ischemia in rats," Neurochemical Research, vol. 36, no. 11, pp. 2022-2028, 2011.

[51] X. Xue, X. J. Qu, Y. Yang et al., "Baicalin attenuates focal cerebral ischemic reperfusion injury through inhibition of nuclear factor $\kappa \mathrm{B}$ p65 activation," Biochemical and Biophysical Research Communications, vol. 403, no. 3-4, pp. 398-404, 2010.

[52] X. K. Tu, W. Z. Yang, S. S. Shi et al., "Baicalin inhibits TLR2/4 signaling pathway in rat brain following permanent cerebral ischemia," Inflammation, vol. 34, no. 5, pp. 463-470, 2011.

[53] X. K. Tu, W. Z. Yang, S. S. Shi, C. H. Wang, and C. M. Chen, "Neuroprotective effect of baicalin in a rat model of permanent focal cerebral ischemia," Neurochemical Research, vol. 34, no. 9, pp. 1626-1634, 2009.

[54] J. Cho and H. K. Lee, "Wogonin inhibits ischemic brain injury in a rat model of permanent middle cerebral artery occlusion," Biological and Pharmaceutical Bulletin, vol. 27, no. 10, pp. 15611564, 2004.

[55] Z. P. Hua, A. J. Shun, S. C. Hyang, J. C. Lee, and W. K. Kim, "Neuroprotective effect of wogonin: potential roles of inflammatory cytokines," Archives of Pharmacal Research, vol. 27, no. 9, pp. 930-936, 2004. 
[56] K. van Leyen, H. Y. Kim, S. R. Lee, G. Jin, K. Arai, and E. H. Lo, "Baicalein and 12/15-lipoxygenase in the ischemic brain," Stroke, vol. 37, no. 12, pp. 3014-3018, 2006.

[57] Y. W. Xu, L. Sun, H. Liang, G. M. Sun, and Y. Cheng, "12/15-Lipoxygenase inhibitor baicalein suppresses PPAR $\gamma$ expression and nuclear translocation induced by cerebral ischemia/reperfusion," Brain Research, vol. 1307, pp. 149-157, 2010.

[58] L. Cui, X. Zhang, R. Yang et al., "Baicalein is neuroprotective in rat MCAO model: role of 12/15-lipoxygenase, mitogen-activated protein kinase and cytosolic phospholipase A2," Pharmacology Biochemistry and Behavior, vol. 96, no. 4, pp. 469-475, 2010.

[59] C. Liu, J. Wu, K. Xu et al., "Neuroprotection by baicalein in ischemic brain injury involves PTEN/AKT pathway," Journal of Neurochemistry, vol. 112, no. 6, pp. 1500-1512, 2010.

[60] P. A. Lapchak, P. Maher, D. Schubert, and J. A. Zivin, "Baicalein, an antioxidant 12/15-lipoxygenase inhibitor improves clinical rating scores following multiple infarct embolic strokes," Neuroscience, vol. 150, no. 3, pp. 585-591, 2007.

[61] S. W. Kim, Y. Jin, J. H. Shin et al., "Glycyrrhizic acid affords robust neuroprotection in the postischemic brain via antiinflammatory effect by inhibiting HMGB1 phosphorylation and secretion," Neurobiology of Disease, vol. 46, no. 1, pp. 147-156, 2012.

[62] T. K. Kao, Y. C. Ou, J. S. Kuo et al., "Neuroprotection by tetramethylpyrazine against ischemic brain injury in rats," Neurochemistry International, vol. 48, no. 3, pp. 166-176, 2006.

[63] S. L. Liao, T. K. Kao, W. Y. Chen et al., "Tetramethylpyrazine reduces ischemic brain injury in rats," Neuroscience Letters, vol. 372, no. 1-2, pp. 40-45, 2004.

[64] T. F. Franke, C. P. Hornik, L. Segev, G. A. Shostak, and C. Sugimoto, "PI3K/Akt and apoptosis: size matters," Oncogene, vol. 22, no. 56, pp. 8983-8998, 2003.

[65] M. P. Scheid, P. A. Marignani, and J. R. Woodgett, "Multiple phosphoinositide 3-kinase-dependent steps in activation of protein kinase B," Molecular and Cellular Biology, vol. 22, no. 17, pp. 6247-6260, 2002.

[66] J. Á. F. Vara, E. Casado, J. de Castro, P. Cejas, C. Belda-Iniesta, and M. González-Barón, "P13K/Akt signalling pathway and cancer," Cancer Treatment Reviews, vol. 30, no. 2, pp. 193-204, 2004.

[67] N. Omori, G. Jin, F. Li et al., "Enhanced phosphorylation of PTEN in rat brain after transient middle cerebral artery occlusion," Brain Research, vol. 954, no. 2, pp. 317-322, 2002.

[68] K. Ning, L. Pei, M. Liao et al., "Dual neuroprotective signaling mediated by downregulating two distinct phosphatase activities of PTEN," Journal of Neuroscience, vol. 24, no. 16, pp. 40524060, 2004.

[69] D. N. Wu, D. S. Pei, Q. Wang, and G. Y. Zhang, "Downregulation of PTEN by sodium orthovanadate inhibits ASK1 activation via PI3-K/Akt during cerebral ischemia in rat hippocampus," Neuroscience Letters, vol. 404, no. 1-2, pp. 98-102, 2006.

[70] J. H. Lee, K. Y. Kim, Y. K. Lee et al., "Cilostazol prevents focal cerebral ischemic injury by enhancing casein kinase 2 phosphorylation and suppression of phosphatase and tensin homolog deleted from chromosome 10 phosphorylation in rats," Journal of Pharmacology and Experimental Therapeutics, vol. 308, no. 3, pp. 896-903, 2004.

[71] T. F. Franke, D. R. Kaplan, and L. C. Cantley, "PI3K: downstream AKTion blocks apoptosis,” Cell, vol. 88, no. 4, pp. 435-437, 1997.
[72] K. Fukunaga and T. Kawano, "Akt is a molecular target for signal transduction therapy in brain ischemic insult," Journal of Pharmacological Sciences, vol. 92, no. 4, pp. 317-327, 2003.

[73] M. Pap and G. M. Cooper, "Role of glycogen synthase kinase-3 in the phosphatidylinositol 3- kinase/Akt cell survival pathway," Journal of Biological Chemistry, vol. 273, no. 32, pp. 19929-19932, 1998.

[74] R. V. Bhat, J. Shanley, M. P. Correll et al., "Regulation and localization of tyrosine216 phosphorylation of glycogen synthase kinase- $3 \beta$ in cellular and animal models of neuronal degeneration," Proceedings of the National Academy of Sciences of the United States of America, vol. 97, no. 20, pp. 11074-11079, 2000.

[75] S. Zimmermann and K. Moelling, "Phosphorylation and regulation of Raf by Akt (protein kinase B)," Science, vol. 286, no. 5445, pp. 1741-1744, 1999. 


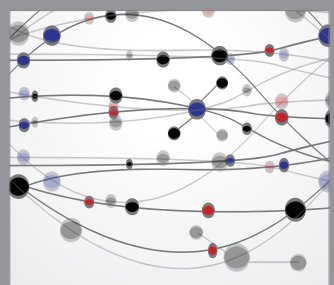

The Scientific World Journal
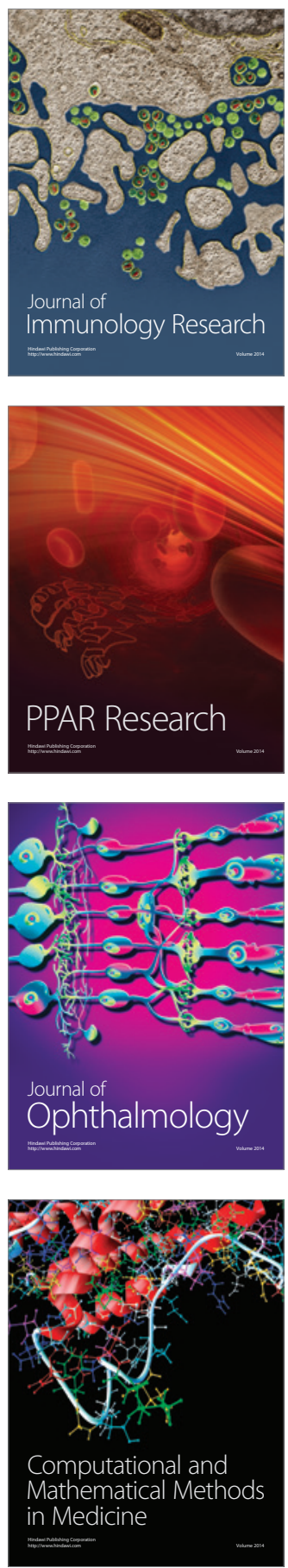

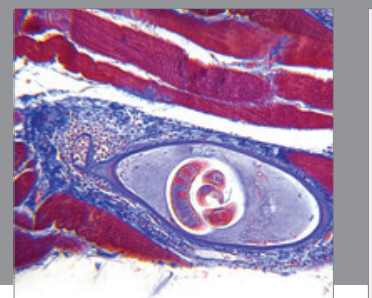

Gastroenterology

Research and Practice
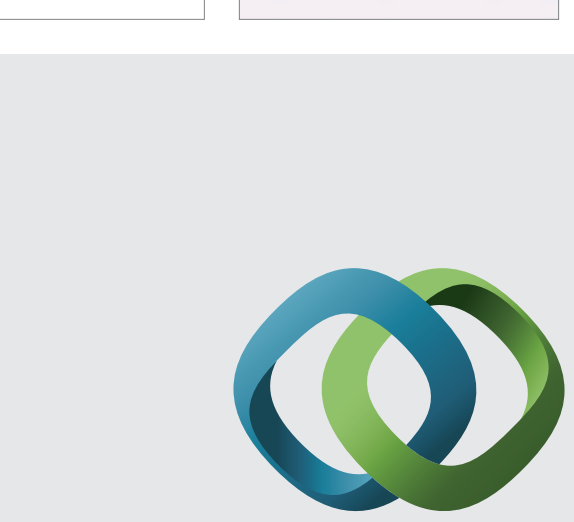

\section{Hindawi}

Submit your manuscripts at

http://www.hindawi.com
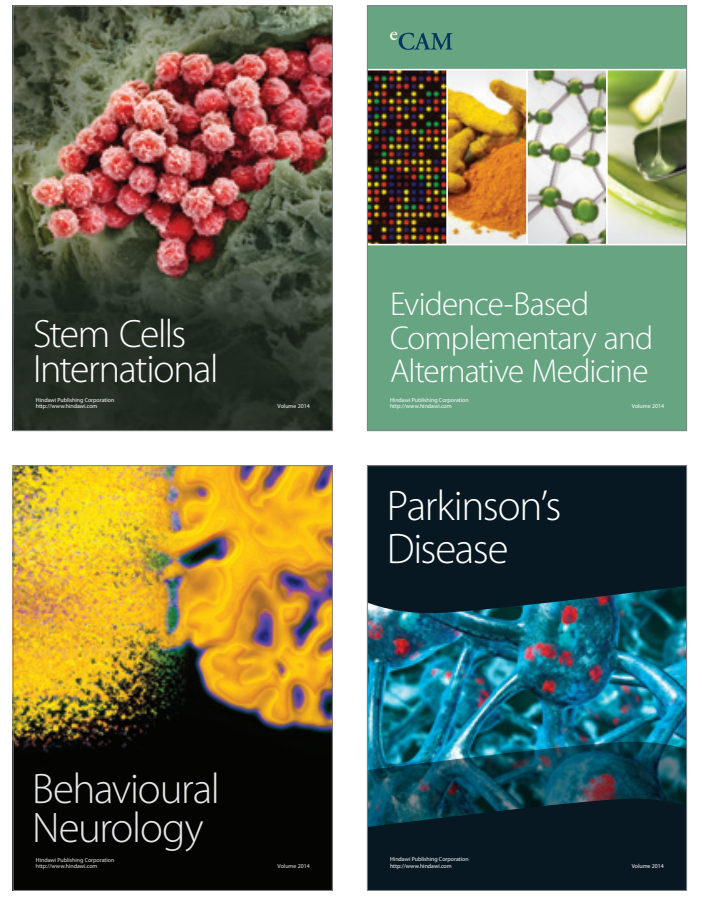
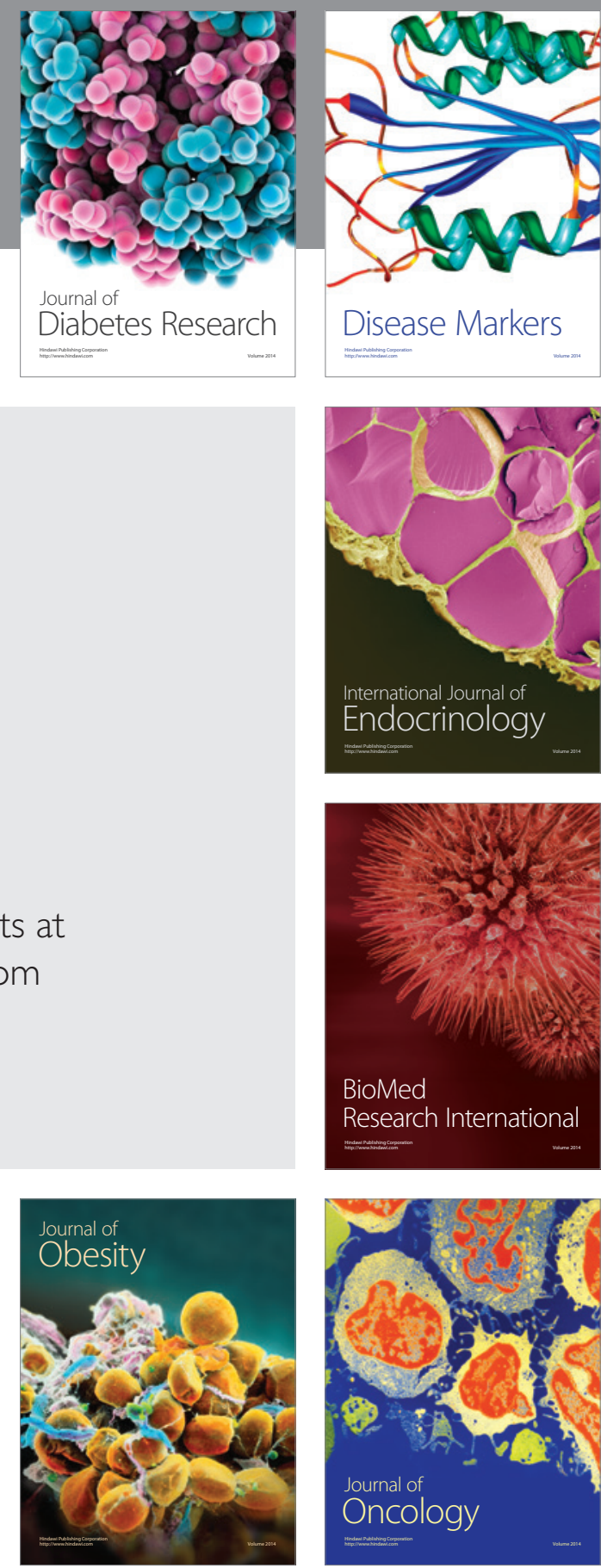

Disease Markers
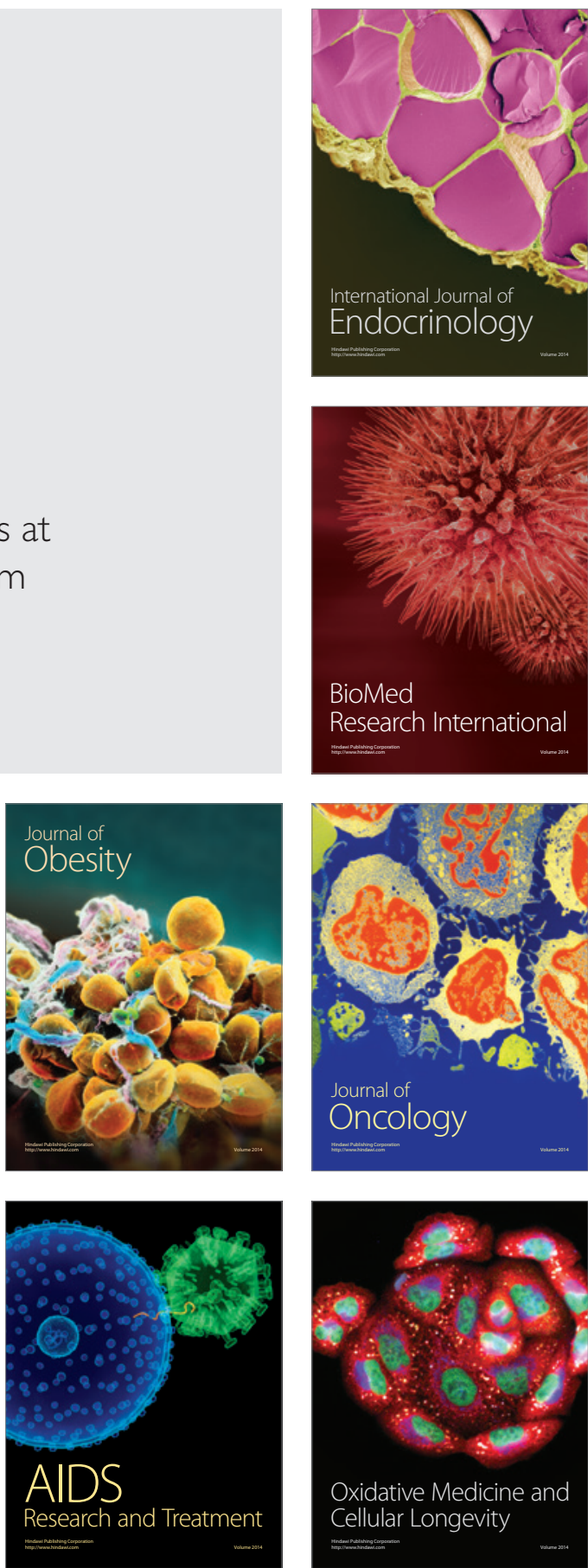